\title{
LYAPUNOV EXPONENTS OF THE HALF-LINE SHE
}

\author{
YIER LIN
}

\begin{abstract}
We consider the half-line stochastic heat equation (SHE) with Robin boundary parameter $A=-\frac{1}{2}$. Under narrow wedge initial condition, we compute every positive (including non-integer) Lyapunov exponents of the half-line SHE. As a consequence, we prove a large deviation principle for the upper tail of the half-line KPZ equation under Neumann boundary parameter $A=-\frac{1}{2}$ with rate function $\Phi_{+}^{\mathrm{hf}}(s)=\frac{2}{3} s^{\frac{3}{2}}$. This confirms the prediction of [KLD18, MV18] for the upper tail exponent of the half-line KPZ equation.
\end{abstract}

\section{INTRODUCTION}

In this paper, we study the half-line KPZ equation, namely the KPZ equation on $\mathbb{R}_{\geq 0}$, with Neumann boundary parameter $A$. Introduced in [CS18], the equation is formally written as

$$
\left\{\begin{array}{l}
\partial_{t} \mathcal{H}^{\mathrm{hf}}(t, x)=\frac{1}{2} \partial_{x x} \mathcal{H}^{\mathrm{hf}}(t, x)+\frac{1}{2}\left(\partial_{x} \mathcal{H}^{\mathrm{hf}}(t, x)\right)^{2}+\xi(t, x), \\
\left.\partial_{x} \mathcal{H}^{\mathrm{hf}}(t, x)\right|_{x=0}=A
\end{array}\right.
$$

where $\xi(t, x)$ is the Gaussian space time white noise. The solution theory of (1.1) is ill-posed due to the nonlinearity and the space-time white noise. One way to properly define the solution is to consider the Hopf-Cole solution $\mathcal{H}^{\mathrm{hf}}(t, x):=\log \mathcal{Z}^{\mathrm{hf}}(t, x)$ where $\mathcal{Z}^{\mathrm{hf}}$ solves the half-line stochastic heat equation (SHE) with Robin boundary parameter $A$, i.e.

$$
\left\{\begin{array}{l}
\partial_{t} \mathcal{Z}^{\mathrm{hf}}(t, x)=\frac{1}{2} \partial_{x x} \mathcal{Z}^{\mathrm{hf}}(t, x)+\frac{1}{2} \mathcal{Z}^{\mathrm{hf}}(t, x) \xi(t, x), \\
\left.\partial_{x} \mathcal{Z}^{\mathrm{hf}}(t, x)\right|_{x=0}=A \mathcal{Z}^{\mathrm{hf}}(t, 0)
\end{array}\right.
$$

We say $\mathcal{Z}^{\text {hf }}$ is a solution to the half-line SHE if for every $t>0, \mathcal{Z}^{\mathrm{hf}}(t, \cdot)$ is adapted to the sigma algebra generated by $\mathcal{Z}^{\text {hf }}(0, \cdot)$ and the space-time white noise up to time $t$, and satisfies the mild formulation

$$
\mathcal{Z}^{\mathrm{hf}}(t, x)=\int_{\mathbb{R}_{\geq 0}} p_{t}^{\mathrm{hf}}(x, y) \mathcal{Z}^{\mathrm{hf}}(0, y) d y+\int_{0}^{t} \int_{\mathbb{R}_{\geq 0}} p_{t-s}^{\mathrm{hf}}(x, y) \mathcal{Z}^{\mathrm{hf}}(s, y) d y d s
$$

For fixed $x \in \mathbb{R}_{\geq 0}, p_{t}^{\mathrm{hf}}(x, y)$ satisfies the half-line heat equation $\partial_{t} p_{t}^{\mathrm{hf}}(x, y)=\frac{1}{2} \partial_{y y} p_{t}^{\mathrm{hf}}(x, y)$ for all $y>0$ with boundary condition $p_{0}^{\mathrm{hf}}(x, y)=\delta_{x}(y)$ and $\partial_{x} p^{\mathrm{hf}}(t, 0)=-A p^{\mathrm{hf}}(t, 0)$. [CS18] proves the existence, uniqueness and positivity of $\mathcal{Z}^{\mathrm{hf}}$ for non-negative boundary parameter $A$ and later [Par19b] extends these results to the scope of all $A \in \mathbb{R}$. As a consequence, the Hopf-Cole solution $\mathcal{H}^{\mathrm{hf}}(t, x)=\log \mathcal{Z}^{\mathrm{hf}}(t, x)$ is well-defined. Note that the solution to (1.1) can also be formulated in other different but equivalent ways, see [GPS17, GH19].

The half-line KPZ equation plays an important role characterizing how the surface grows subject to a boundary. In addition, it is the (weak) scaling limit of various half space models lying in the half-space KPZ universality [Wu18, CS18, Par19a]. Interestingly, such half-space random growth models usually exhibit a phase transition depending on the strength of repulsion/attraction at the boundary, which is characterized by the boundary parameter. Such phase transition is related to wetting/depinning transition which goes back to [Kar85, Kar87] and was proved for various discrete half-space models [BR01, SI04, BBCS18].

For the half-line KPZ equation, we restrict ourselves in a particular initial condition called narrow wedge initial condition, which corresponds to setting $\mathcal{Z}^{\mathrm{hf}}(0, x)$ to be a Dirac-delta function at zero. It is widely believed that the fluctuation of $\mathcal{H}^{\mathrm{hf}}(2 t, 0)$ at late time exhibits a phase transition at $A=-\frac{1}{2}$ [Par19b, Conjecture 1.2]. More precisely, the fluctuation of $\mathcal{H}^{\mathrm{hf}}(2 t, 0)$ will be Gaussian/Tracy-Widom GOE/GSE [TW96] depending on whether the boundary parameter $A$ is smaller than, equal to or larger than $-\frac{1}{2}$. 
[Par19b, Theorem 1.1] (also see [BBCW18, Remark 1.1]) shows that when $A=-\frac{1}{2}$, for all $s \in \mathbb{R}$,

$$
\lim _{t \rightarrow \infty} \mathbb{P}\left(\frac{\mathcal{H}^{\mathrm{hf}}(2 t, 0)+\frac{t}{12}}{t^{\frac{1}{3}}} \leq s\right)=F_{\mathrm{GOE}}(s),
$$

where $F_{\mathrm{GOE}}(s)$ is distribution function of the Tracy-Widom GOE distribution [TW96]. The key ingredient to arriving at (1.2) is the exact formula for the Laplace transform of $\mathcal{Z}^{\mathrm{hf}}(2 t, 0)+\frac{t}{12}$ developed in [BBCW18, Par19b], see Theorem 1.3. The conjectured Gaussian/GSE fluctuation for $A<-\frac{1}{2}$ and $A>-\frac{1}{2}$ are supported in a nonrigorous way by the works [GLD12, BBC16, DNKDT19, KLD20]. In the GSE region $A>-\frac{1}{2}$, an exact formula of the Laplace transform of $\mathcal{Z}^{\text {hf }}(2 t, 0)$ is also conjectured in the aforementioned works.

In this paper, we focus on the critical regime $A=-\frac{1}{2}$.

Having considered the limit theorem (1.2), it is natural to think about the large deviation principle (LDP), i.e. the probability that $\mathcal{H}^{\mathrm{hf}}(2 t, 0)+\frac{t}{12}$ deviates from zero in a size of $t$, as $t \rightarrow \infty$. It is expected that for $s>0$,

$$
\begin{aligned}
& -\lim _{t \rightarrow \infty} \frac{1}{t^{2}} \log \mathbb{P}\left(\mathcal{H}^{\mathrm{hf}}(2 t, 0)+\frac{t}{12}<-s t\right)=\Phi_{-}^{\mathrm{hf}}(s) \\
& -\lim _{t \rightarrow \infty} \frac{1}{t} \log \mathbb{P}\left(\mathcal{H}^{\mathrm{hf}}(2 t, 0)+\frac{t}{12}>s t\right)=\Phi_{+}^{\mathrm{hf}}(s) .
\end{aligned}
$$

(upper tail)

Note that the upper and lower tail LDP have different speeds $\left(t\right.$ vs $\left.t^{2}\right)$. One way to explain such phenomenon is to view $\mathcal{H}^{\text {hf }}(2 t, 0)$ as the free energy of a half-space continuum directed random polymer with a wall at $x=0$. For various discrete/continuum polymers, the $t$ vs $t^{2}$ phenomenon is observed and explained in [LDMS16, BGS17, DT19]. Here, let we provide a different explanation. If we replace $s$ with $t^{\frac{2}{3}} s$ in (1.2), the right hand side of $(1.2)$ becomes $F_{\mathrm{GOE}}\left(t^{\frac{2}{3}} \mathrm{~s}\right)$. Since Tracy-Widom GOE distribution has left and right tail: as $s \rightarrow \infty F_{\mathrm{GOE}}(-s) \sim \exp \left(-\frac{s^{3}}{24}\right), 1-F_{\mathrm{GOE}}(s) \sim \exp \left(-\frac{2}{3} s^{\frac{3}{2}}\right)$, see [TW09]. Hence, we recover the $t^{2}$ and $t$ speed of LDP for the lower and upper tail. [Tsa18, Corollary 1.3] proves the LDP for the lower tail and identifies the rate function $\Phi_{-}^{\mathrm{hf}}(s)$.

In this paper, we prove that the upper tail LDP holds with $\Phi_{+}^{\mathrm{hf}}(s)=\frac{2}{3} s^{\frac{3}{2}}$. Note that this is the first rigorous result concerning the upper tail LDP of the half-space models in the KPZ universality class. The $\frac{3}{2}$-exponent for the upper tail also arises in the work of [KLD18, MV18], where the LDP for half-line KPZ equation at short time was studied.

The upper tail LDP of the half-line KPZ equation is closely related to the Lyapunov exponent of the half-line SHE. More precisely, for $p \in \mathbb{R}_{>0}$ we call the $p$-th Lyapunov exponent of the SHE to be limit of $t^{-1} \log \mathbb{E}\left[\mathcal{Z}^{\text {hf }}(2 t, 0)^{p}\right]$ as $t \rightarrow \infty$. If such limit exists for every $p$, in the spirit of Gärtner-Ellis theorem, $\mathcal{H}^{\mathrm{hf}}(2 t, 0)=\log \mathcal{Z}^{\mathrm{hf}}(2 t, 0)$ satisfies a LDP with rate function to be the Legendre-Fenchel transform of the Lyapunov exponents (as a function of $p$ ). We remark that the Lyapunov exponents also capture the nature of intermittency, which is a universal property for the random fields with multiplicative noise and has been studied extensive in the literature [GM90, CCM94, GKM07, FK09, CJK13, CJKS13, CD15, Che15, BC16, KKX17].

1.1. Main result and proof idea. From now on, we use $\mathcal{Z}^{\text {hf }}(2 t, 0)$ to denote the solution to the half-line SHE with Robin boundary parameter $A=-\frac{1}{2}$ and Dirac-delta initial data $\mathcal{Z}^{\text {hf }}(0, x)=\delta_{x=0}$. Our main contribution is rigorously computing the Lyapunov exponents of the half-line SHE.

Theorem 1.1 (Lyapunov exponents and upper tail LDP). We have

(i) For every $p \in \mathbb{R}_{>0}$, one has $\lim _{t \rightarrow \infty} \frac{1}{t} \log \mathbb{E}\left[\mathcal{Z}^{h f}(2 t, 0)^{p} \exp \left(\frac{p t}{12}\right)\right]=\frac{p^{3}}{3}$.

(ii) For every $s \in \mathbb{R}_{>0}$, one has the upper tail $L D P:-\lim _{t \rightarrow \infty} \frac{1}{t} \log \mathbb{P}\left(\mathcal{H}^{h f}(2 t, 0)+\frac{t}{12}>s t\right)=\Phi_{+}^{h f}(s)=\frac{2}{3} s^{\frac{3}{2}}$.

Remark 1.2. The above upper tail LDP rate function matches with the right tail of the GOE, which is the limiting distribution in (1.2). Such matching between the upper tail LDP rate function of KPZ equation and the right tail of the limiting Tracy GUE/GOE/Baik-Rains distribution has been predicted in [LDMS16, LDMRS16, MS17] and has been confirmed in various situations [DT19, GL20].

Let us briefly explain the idea for the proof of Theorem 1.1. A more detailed discussion will be given in Section 2. First of all, it is not hard to get Theorem 1.1 (ii) once we obtain (i), see Proposition 1.12 of [GL20]. Hence, we focus on the proof of (i). One crucial input is the following exact formula obtained in [Par19b, Theorem 1.3] (also see [BBCW18, Theorem 7.6]), which is stated as follows. 
Theorem 1.3 (Theorem 1.3 of [Par19b], Theorem 7.6 of [BBCW18]). When $A=-\frac{1}{2}$, we have for all $s \geq 0$,

$$
\mathbb{E}\left[\exp \left(-s \mathcal{Z}^{h f}(2 t, 0) e^{\frac{t}{12}}\right)\right]=\mathbb{E}\left[\prod_{i=1}^{\infty} \frac{1}{\sqrt{1+4 s \exp \left(t^{\frac{1}{3}} \mathbf{a}_{\mathbf{k}}\right)}}\right],
$$

where $\mathbf{a}_{1}>\mathbf{a}_{2}>\cdots$ is the GOE-Airy point process defined in Definition 2.1.

Our argument for proving Theorem 1.1 (i) follows [DT19] at the beginning. Firstly, we write the $p$-th moment of $\mathcal{Z}^{\mathrm{hf}}(2 t, 0) e^{\frac{t}{12}}$ in terms of the Laplace transform (Lemma 2.5)

$$
\mathbb{E}\left[\left(\mathcal{Z}^{\mathrm{hf}}(2 t, 0) e^{\frac{t}{12}}\right)^{p}\right]=\frac{(-1)^{n}}{\Gamma(1-\alpha)} \int_{0}^{\infty} s^{-\alpha} \partial_{s}^{n}\left(\mathbb{E}\left[\exp \left(-s \mathcal{Z}^{\mathrm{hf}}(2 t, 0) e^{\frac{t}{12}}\right)\right]\right) d s, \quad n=\lfloor p\rfloor+1, \alpha=p+1-n .
$$

Decompose the integral region into $(0,1]$ and $(1, \infty)$ and denote the latter integral by $\mathcal{R}_{p}(t)$, we get

$$
\mathbb{E}\left[\left(\mathcal{Z}^{\mathrm{hf}}(2 t, 0) e^{\frac{t}{12}}\right)^{p}\right]=\frac{(-1)^{n}}{\Gamma(1-\alpha)} \int_{0}^{1} s^{-\alpha} \partial_{s}^{n}\left(\mathbb{E}\left[\exp \left(-s \mathcal{Z}^{\mathrm{hf}}(2 t, 0) e^{\frac{t}{12}}\right)\right]\right) d s+\mathcal{R}_{p}(t)
$$

It turns out $\mathcal{R}_{p}(t)$ is uniformly bounded in $t$ so we only need to focus on the first term on the right hand side (1.4). The laplace transform $\mathbb{E}\left[\exp \left(-s \mathcal{Z}^{\mathrm{hf}}(2 t, 0) e^{\frac{t}{12}}\right)\right]$ in the above integral admits an explicit formula in terms of the GOE point process given by the right hand side of (1.3). Rewrite the expectation of products of the GOE point process into a Fredholm Pfaffian (Lemma 2.4), we get

$$
\mathbb{E}\left[\exp \left(-s \mathcal{Z}^{\mathrm{hf}}(2 t, 0)\right) e^{\frac{t}{12}}\right]=1+\sum_{L=1}^{\infty} \frac{1}{L !} \int_{\mathbb{R}^{L}} \operatorname{Pf}\left[K\left(x_{i}, x_{j}\right)\right]_{i, j=1}^{L} \prod_{i=1}^{L} \phi_{s, t}\left(x_{i}\right) d x_{i},
$$

where the Pfaffian kernel $K$ is $2 \times 2$ matrix defined in Definition 2.1 and the function $\phi_{s, t}$ is specified in (2.5). Inserting the above expression to the first term on the right hand side of (1.4), bringing the infinite summation over $L$ outside the derivative over $s$ and integral from 0 to 1 (which will be justified), we get

$$
\mathbb{E}\left[\left(\mathcal{Z}^{\mathrm{hf}}(2 t, 0) e^{\frac{t}{12}}\right)^{p}\right]=\sum_{L=1}^{\infty} \frac{(-1)^{n}}{\Gamma(1-\alpha) L !} \int_{0}^{1} s^{-\alpha} \int_{\mathbb{R}^{L}} \operatorname{Pf}\left[K\left(x_{i}, x_{j}\right)\right]_{i, j=1}^{L} \partial_{s}^{n}\left(\prod_{i=1}^{L} \phi_{s, t}\left(x_{i}\right)\right) d x_{1} \ldots d x_{L}+\mathcal{R}_{p}(t) .
$$

The next step is to decompose the infinite summation in the above display into $L=1$ and $L \geq 2$. In particular, we denote the first term in the summation by $\mathcal{A}_{p}(t)$ and the $L$-th term $(L \geq 2)$ by $\mathcal{B}_{p, L}(t)$, then

$$
\mathbb{E}\left[\left(\mathcal{Z}^{\mathrm{hf}}(2 t, 0) e^{\frac{t}{12}}\right)^{p}\right]=\mathcal{A}_{p}(t)+\sum_{L=2}^{\infty} \mathcal{B}_{p, L}(t)+\mathcal{R}_{p}(t)
$$

We call $\mathcal{A}_{p}(t)$ the leading order term which will be shown to hold the dominating $t \rightarrow \infty$ asymptotic. In particular, it equals an integral of the $(1,2)$ entry of the $2 \times 2$ matrix $K(x, x)$ denoted by $K_{12}(x, x)$. The second term on the right hand side of the above display is composed of the higher order terms. Each $\mathcal{B}_{p, L}(t)$ is related to an integral of $L$-th correlation function $\operatorname{Pf}\left[K\left(x_{i}, x_{j}\right)\right]_{i, j=1}^{L}$ of the GOE point process. In our proof, we show in Proposition 2.8 and 2.9 that for fixed $p>0$, as $t \rightarrow \infty, 1): \mathcal{A}_{p}(t)$ grows asymptotically as $\exp \left(p^{3} t / 3\right)$. 2) : $\sum_{L=2}^{\infty}\left|\mathcal{B}_{p, L}(t)\right|$ is upper bounded by $\exp \left(\left(p^{3}-\delta_{p}\right) t / 3\right)$ for some $\delta_{p}>0$. This demonstrates Theorem 1.1 (i).

So far, we have followed the idea in [DT19]. The analysis of the leading order term $\mathcal{A}_{p}(t)$ in Proposition 2.8 involves a steepest descent type analysis of the integral of $K_{12}(x, x)$. The harder problem is to control the higher order terms. In the situation of [DT19], the authors deal with a Fredholm determinant $\operatorname{det}(I+A)=$ $1+\sum_{L=1}^{\infty} \operatorname{Tr}\left(A^{\wedge L}\right),{ }^{1}$ where $A$ is a positive, trace class operator. [DT19] upper bounds the higher order term $\operatorname{Tr}\left(A^{\wedge L}\right)$ by $(\operatorname{Tr}(A))^{L} / L$ !. This can be understood by setting $\left\{\lambda_{i}\right\}_{i=1}^{\infty}$ to be the eigenvalues of $A$, since $\lambda_{i}$ are all non-negative,

$$
\operatorname{Tr}\left(A^{\wedge L}\right)=\sum_{1 \leq i_{1}<\cdots<i_{L}} \lambda_{i_{1}} \ldots \lambda_{i_{L}} \leq \frac{1}{L !}\left(\sum_{i=1}^{\infty} \lambda_{i}\right)^{L}=\frac{1}{L !}(\operatorname{Tr}(A))^{L} .
$$

Unfortunately, we are unaware of an analogue for such bound in the case of Fredholm Pfaffian. Instead of mimicking [DT19], we adopt a more direct approach. Using Hadamard's inequality and a determinantal analysis, we obtain two upper bounds of $\operatorname{Pf}\left[K\left(x_{i}, x_{j}\right)\right]_{i, j=1}^{L}$ (Proposition 4.1). These upper bounds will be applied to control various terms in $\sum_{L=2}^{\infty} \mathcal{B}_{p, L}(t)$ depending on whether $L$ is greater than a fixed threshold. We want to

\footnotetext{
${ }^{1}$ There is a misstatement in page 4 of [DT19] where an extra $L$ ! appears in the definition of Fredholm determinant.
} 
highlight that we did not pursue to get the sharp bound of the $p$-th moment of $\mathcal{Z}^{\text {hf }}(2 t, 0)$ which holds uniformly for large $p$ and $t$, as shown in [DT19, Theorem $1.1(\mathrm{a})^{*}$ ]. However, it is sufficient to apply our method to obtain the Lyapunov exponents and LDP for our problem as well as for [DT19, Theorem 1.1].

1.2. Previous results. Recently, there has been significant progress in understanding the Lyapunov exponents and tails of various Stochastic PDEs, see [GL20, Section 1.2] and reference therein. Here, we restrict our discussion to the scope of the KPZ equation and the SHE.

1.2.1. Full line KPZ equation/SHE. The KPZ equation was introduced in [KPZ86] as a paradigmatic model for the random surface growth. It is a representative of the $K P Z$ universality class [ACQ11, Cor12], a collection of models sharing the universal scaling exponent and large time scaling behavior. Recently, the upper/lower tail LDP of the KPZ equation receives plenty of attention from the mathematics and physics community. In fact, there are two regimes for the LDP of the KPZ equation, long time and short time (Freidlin-Wentzell regime). We will focus on discussing the long time regime and for the latter situation, see the physics literature [KK07, KK09, KMS16, MKV16, LDMRS16].

The upper tail of the KPZ equation is closely connected to the Lyapunov exponents of SHE. [BC95] first computes the integer Lyapunov exponents of the SHE. However, due to an incorrect use of Skorokhod's lemma, their result is only valid for the second moment. By analyzing the Brownian local time from Feynman-Kac representation of the SHE, [Che15] obtained the integer Lyapunov exponents for the SHE under deterministic bounded initial data. For the SHE under narrow wedge initial condition, the integer moment of the solution admits a contour integral formula [BC14, Gho18]. By a residue calculus, [CG20a] obtains the integer Lyapunov exponents, from which they obtain a bound for the upper tail of the KPZ equation, showing the correct exponent 3/2. [DT19] improves their result by identifying all positive real Lyapunov exponents of the SHE. As a consequence, they obtain the upper tail LDP of the KPZ equation with rate function $\frac{4}{3} s^{\frac{3}{2}}$. Recently, [GL20] is capable of computing all positive Lyapunov exponents for the SHE starting with a class of general (including random) initial data and obtain the corresponding upper tail LDP of the KPZ equation.

Unlike the upper tail, the lower tail of the KPZ equation does not have a strong connection to the moment of SHE. For the narrow wedge initial condition, via a delicate analysis of the exact formula in [BG16], [CG20b] derives a tight bound which detects the crossover of the tail exponent from 3 to $5 / 2$ depending on the depth of the tail, which was first observed in the physics work [SMP17]. The LDP for the lower tail of the KPZ equation is obtained by [Tsa18, CC19]. For the KPZ equation with general initial data, [CG20b] obtains an upper bound for the lower tail probability. Besides that, very few things are known at present.

1.2.2. Half-line $K P Z$ equation/SHE. Compared with the knowledge for full-line equation, smaller amount of results are known for the half-line KPZ equation/SHE. [CS18] proves that on a closed interval or a half line, the open ASEP weakly converges to the KPZ equation with Neumann boundary parameter $A \geq 0$. Such convergence was extended later by [Par19b] to all $A \in \mathbb{R}$. [BBCW18, Par19b] obtain the Laplace transform formula for the half-line SHE under narrow wedge initial condition when $A=-\frac{1}{2}$, which helps to capture the Tracy-Widom GOE fluctuation of the KPZ equation. As discussed before, there is a conjectured Gaussian-GOE-GSE phase transition the for half-line KPZ equation, which is only proved at the critical parameter $A=-\frac{1}{2}$. Recently, there are progress identifying new limiting distribution for the half-space KPZ equation under stationary initial data [BKD20]. Such distribution is believed to be universal and arises in other half-space model starting from stationary initial data [BFO20].

Regarding the tail of half-space KPZ equation, let us focus on $A=-\frac{1}{2}$ and narrow wedge initial condition. Results for other boundary parameters and initial conditions are fairly untouched for now. Under the aforementioned boundary condition, a tight estimate of the lower tail was obtained in [Kim19], which detects the similar crossover of the tail exponent that appears in the full-line situation. The LDP for the lower tail was obtained by [Tsa18]. Few things were rigorously proved for the upper tail aside from the current work. [BBC16] (also see [BBC20]) obtains a moment formula of the half-line SHE by solving the delta-Bose gas (the result is not rigorous, since the uniqueness of the solution to the delta-Bose gas is unknown). It is also unclear whether one can extract the integer Lyapunov exponents for the half-line SHE (thus obtaining tail bounds of the half-line $\mathrm{KPZ}$ equation) from a similar residue calculus of the integral formula as carried out in [CG20a], due to the extra complexity.

On a different aspect, it is worth to mention the works of [KLD18, MV18] in which the authors consider the 
LDP for the half-line KPZ equation in short time. The same exponent $3 / 2$ in the rate function is obtained therein. In addition, [KLD18] predicted the upper tail exponent to be $\frac{2}{3} x^{\frac{3}{2}}$ when $A=-\frac{1}{2}, 0$ and $\frac{4}{3} x^{\frac{3}{2}}$ when $A=+\infty$. For the future work, it is appealing to prove a LDP for the upper tail for general boundary parameter $A$ and see how the LDP rate function changes when $A$ belongs to the Gaussian/GSE regime.

Outline. The rest of the paper is organized as follows. In section 2, we give an overview for the proof of the main theorem and provide more details for what is discussed in Section 1.1. In particular, we transform our problem into proving Proposition 2.8 and 2.9. Section 3 was devoted to prove Proposition 2.8. In Section 4, we provide two different upper bound for the pfaffian $\operatorname{Pf}\left[K\left(x_{i}, x_{j}\right)\right]_{i, j=1}^{L}$ which is crucial to the proof of Proposition 2.9. We also justify Lemma 2.7 in that section. Section 5 completes the proof of Proposition 2.9 .

Acknowledgment. The author thanks Guillaume Barraquand, Ivan Corwin, Sayan Das and Li-Cheng Tsai for helpful discussions. The author was partially supported by the Fernholz Foundation's "Summer Minerva Fellow" program and also received summer support from Ivan Corwin's NSF grant DMS-1811143, DMS-1664650.

\section{Proof of Theorem 1.1: A Detailed overview}

In this section, we explain with more details how we arrive at proving Theorem 1.1. We begin with the definition of GOE point process that was mentioned in the introduction. As formulated in [AGZ10, Section 4.2.1], a point process on $\mathbb{R}$ is a random point configuration $\mathcal{X}$. The $L$-th correlation function $\rho_{L}$ w.r.t. the measure $\mu$ associated to $\mathcal{X}$ is defined in the way that for arbitrary distinct Borel sets $B_{1}, \ldots, B_{L}$,

$$
\int_{B_{1} \times \cdots \times B_{L}} \rho_{L}\left(x_{1}, \ldots, x_{L}\right) d \mu^{\otimes L}=\mathbb{E}\left[\#\left\{\left(x_{1}, \ldots, x_{L}\right), \text { such that } x_{i} \in \mathcal{X} \cap B_{i}, i=1, \ldots, L\right\}\right] .
$$

We say a point process is a Pfaffian, if there exists a matrix kernel $K: \mathbb{R} \times \mathbb{R} \rightarrow \mathbb{C}^{2 \times 2}$ such that the correlation function $\rho_{L}\left(x_{1}, \ldots, x_{L}\right)=\operatorname{Pf}\left[K\left(x_{i}, x_{j}\right)\right]_{i, j=1}^{L}$ for arbitrary $L \in \mathbb{Z}_{\geq 1}$.

Definition 2.1 (GOE point process). We say $\mathcal{X}=\left\{\mathbf{a}_{1}>\mathbf{a}_{2}>\cdots\right\}$ is the GOE-Airy point process, if it is a Pfaffian point process on $\mathbb{R}$ with kernel

$$
K(x, y)=\left[\begin{array}{ll}
K_{11}(x, y), & K_{12}(x, y) \\
K_{21}(x, y), & K_{22}(x, y)
\end{array}\right]
$$

with the entries

$$
\begin{aligned}
K_{11}(x, y)= & \int_{0}^{\infty} \operatorname{Ai}(x+\lambda) \operatorname{Ai}^{\prime}(y+\lambda)-\operatorname{Ai}(y+\lambda) \operatorname{Ai}^{\prime}(x+\lambda) d \lambda \\
K_{12}(x, y)= & -K_{21}(y, x)=\frac{1}{2} \int_{0}^{\infty} \operatorname{Ai}(x+\lambda) \operatorname{Ai}(y+\lambda) d \lambda+\frac{1}{2} \operatorname{Ai}(x) \int_{-\infty}^{y} \operatorname{Ai}(\lambda) d \lambda, \\
K_{22}(x, y)= & \frac{1}{4} \int_{0}^{\infty}\left(\int_{\lambda}^{\infty} \operatorname{Ai}(y+\mu) d \mu\right) \operatorname{Ai}(x+\lambda) d \lambda-\frac{1}{4} \int_{0}^{\infty}\left(\int_{\lambda}^{\infty} \operatorname{Ai}(x+\mu) d \mu\right) \operatorname{Ai}(y+\lambda) d \lambda \\
& -\frac{1}{4} \int_{0}^{\infty} \operatorname{Ai}(x+\lambda) d \lambda+\frac{1}{4} \int_{0}^{\infty} \operatorname{Ai}(y+\lambda) d \lambda-\frac{\operatorname{sgn}(x-y)}{4} .
\end{aligned}
$$

Here, $\operatorname{sgn}(x)$ is defined as the sign function $\mathbf{1}_{\{x>0\}}-\mathbf{1}_{\{x<0\}}$. Furthermore, we set $K_{21}(x, y)=-K_{12}(y, x)$.

Remark 2.2. Note that our expression of the kernel (2.1), (2.2), (2.3) is different from that in Eq. (6.1a), (6.1b), (6.1c) of [BBCW18]. However, they are demonstrated to be the same, see (2.9) and (6.17) of [Fer04] or [BBCS18, Lemma 2.6].

It turns out that the right hand side of (1.3) can be rewritten as a Fredholm Pfaffian, which has been first defined in [Rai00]. We reproduce the definition of the Fredholm Pfaffian from [BBCS18, Definition 2.3].

Definition 2.3 (Fredholm Pfaffian). Let $K(x, y)$ be an asymmetric $2 \times 2$ matrix and $\mu$ to be a measure on $\mathbb{R}$ and $f: \mathbb{R} \rightarrow \mathbb{C}$ be a measurable function. We define the Fredholm Pfaffian by the series expansion

$$
\operatorname{Pf}[J+K]_{L^{2}(\mathbb{R}, f \mu)}=\sum_{L=0}^{\infty} \frac{1}{L !} \int_{\mathbb{R}^{L}} \operatorname{Pf}\left[K\left(x_{i}, x_{j}\right)\right]_{i, j=1}^{L}\left(\prod_{i=1}^{L} f\left(x_{i}\right)\right) d \mu^{\otimes L}\left(x_{1}, \ldots, x_{L}\right),
$$

where

$$
J(x, y)=\mathbf{1}_{\{x=y\}}\left[\begin{array}{cc}
0 & 1 \\
-1 & 0
\end{array}\right] .
$$


Lemma 2.4 (Pfaffian point process and Fredholm Pfaffian [Rai00]). Let $\mathbf{a}_{\mathbf{1}}>\mathbf{a}_{2}>\ldots$ be a Pfaffian point process with kernel $K$ and $f: \mathbb{R} \rightarrow \mathbb{C}$ be a measurable function. We have

$$
\mathbb{E}\left[\prod_{i=1}^{\infty}\left(1+f\left(\mathbf{a}_{i}\right)\right)\right]=\operatorname{Pf}[J+K]_{L^{2}(\mathbb{R}, f \mu)},
$$

as long as both sides of the above equation converge absolutely.

We take $\mathbf{a}_{1}>\mathbf{a}_{2}>\ldots$ to be the GOE point process defined in Definition 2.1 and the function $f$ in the above lemma to be

$$
\phi_{s, t}(x):=\frac{1}{\sqrt{1+4 s \exp \left(t^{1 / 3} x\right)}}-1,
$$

Theorem 7.6 of [BBCW18] has already justified the convergence of both sides of (2.4). As a result,

$$
\mathbb{E}\left[\prod_{i=1}^{\infty} \frac{1}{\sqrt{1+4 s \exp \left(t^{\frac{1}{3}} \mathbf{a}_{i}\right)}}\right]=\sum_{L=0}^{\infty} \frac{1}{L !} \int_{\mathbb{R}^{L}} \operatorname{Pf}\left[K\left(x_{i}, x_{j}\right)\right]_{i, j=1}^{L} \prod_{i=1}^{L} \phi_{s, t}\left(x_{i}\right) d x_{i} .
$$

By Theorem 1.3, the left hand side in the above display equals the Laplace transform of $\mathcal{Z}^{\text {hf }}(2 t, 0) \exp \left(\frac{t}{12}\right)$, thus

$$
\mathbb{E}\left[\exp \left(-s \mathcal{Z}^{\mathrm{hf}}(2 t, 0) e^{\frac{t}{12}}\right)\right]=\sum_{L=0}^{\infty} \frac{1}{L !} \int_{\mathbb{R}^{L}} \operatorname{Pf}\left[K\left(x_{i}, x_{j}\right)\right]_{i, j=1}^{L} \prod_{i=1}^{L} \phi_{s, t}\left(x_{i}\right) d x_{i}
$$

To prove Theorem 1.1, the next step is to link the Laplace transform of $\mathcal{Z}^{\text {hf }}(2 t, 0) \exp \left(\frac{t}{12}\right)$ with its fractional moment. The following lemma was stated as [DT19, Lemma 1.2], which can be verified via Fubini's theorem.

Lemma 2.5. For arbitrary non-negative random variable $X, 0 \leq \alpha<1$ and $n \in \mathbb{Z}_{\geq 1}$,

$$
\mathbb{E}\left[X^{n-1+\alpha}\right]=\frac{(-1)^{n}}{\Gamma(1-\alpha)} \int_{0}^{\infty} s^{-\alpha} \partial_{s}^{n} \mathbb{E}\left[e^{-s X}\right] d s .
$$

As a convention, we use $\partial_{s}^{n} \mathbb{E}\left[e^{-s X}\right]$ to denote the $n$-th derivative of $\mathbb{E}\left[e^{-s X}\right]$ with respect to $s$.

For fixed $p>0$, we set $n=\lfloor p\rfloor+1$ and $\alpha=p-n+1$. It is clear that $n \in \mathbb{Z}_{\geq 1}, \alpha \in[0,1)$. Applying Lemma 2.5 with $X=\mathcal{Z}^{\mathrm{hf}}(2 t, 0) \exp \left(\frac{t}{12}\right)$ (note that $\mathcal{Z}^{\mathrm{hf}}(2 t, 0)$ is almost surely positive), we find that

$$
\mathbb{E}\left[\mathcal{Z}^{\mathrm{hf}}(2 t, 0)^{p} e^{\frac{p t}{12}}\right]=\frac{(-1)^{n}}{\Gamma(1-\alpha)} \int_{0}^{\infty} s^{-\alpha} \partial_{s}^{n} \mathbb{E}\left[e^{-s\left(\mathcal{Z}^{\mathrm{hf}}(2 t, 0)+\frac{t}{12}\right)}\right] d s .
$$

Splitting the interval of integration into $[0,1]$ and $[1, \infty)$ yields

$$
\mathbb{E}\left[\mathcal{Z}^{\mathrm{hf}}(2 t, 0)^{p} e^{\frac{p t}{12}}\right]=\frac{(-1)^{n}}{\Gamma(1-\alpha)} \int_{0}^{1} s^{-\alpha} \partial_{s}^{n} \mathbb{E}\left[e^{-s\left(\mathcal{Z}^{\mathrm{hf}}(2 t, 0)+\frac{t}{12}\right)}\right] d s+\mathcal{R}_{p}(t),
$$

where $\mathcal{R}_{p}(t):=\frac{(-1)^{n}}{\Gamma(1-\alpha)} \int_{1}^{\infty} s^{-\alpha} \partial_{s}^{n} \mathbb{E}\left[e^{-s\left(\mathcal{Z}^{\mathrm{hf}}(2 t, 0)+\frac{t}{12}\right)}\right] d s$.

Lemma 2.6. For fixed $p>0,\left|\mathcal{R}_{p}(t)\right|$ is uniformly bounded by a constant for every $t>0$.

Proof. Since $\mathcal{R}_{p}(t)=\frac{(-1)^{n}}{\Gamma(1-\alpha)} \int_{1}^{\infty} s^{-\alpha} \mathbb{E}\left[e^{-s X} X^{n}\right] d s$ with $X=\mathcal{Z}^{\text {hf }}(2 t, 0) \exp \left(\frac{t}{12}\right)$. Note that

$$
\mathbb{E}\left[e^{-s X} X^{n}\right] \leq \sup _{x \geq 0}\left(e^{-s x} x^{n}\right)=s^{-n} n^{n} e^{-n} .
$$

Replacing $\mathbb{E}\left[e^{-s X} X^{n}\right]$ with this upper bound inside the integral yields

$$
0 \leq(-1)^{n} \mathcal{R}_{p}(t) \leq \frac{1}{\Gamma(1-\alpha)} \int_{1}^{\infty} s^{-n-\alpha} n^{n} e^{-n} d s=\frac{n^{n} e^{-n}}{\Gamma(1-\alpha)(n+\alpha)} .
$$

Since $\alpha$ and $n$ are determined by $p$, so the right hand side is a constant that only depends on $p$, this completes our proof.

By (2.6), we see that the first term on the RHS of (2.7) can be written as

$$
\int_{0}^{1} s^{-\alpha} \partial_{s}^{n} \mathbb{E}\left[e^{-s\left(\mathcal{Z}^{\mathrm{hf}}(2 t, 0)+\frac{t}{12}\right)}\right] d s=\int_{0}^{1} s^{-\alpha} \partial_{s}^{n}\left(\sum_{L=1}^{\infty} \frac{1}{L !} \int_{\mathbb{R}^{L}} \operatorname{Pf}\left[K\left(x_{i}, x_{j}\right)\right]_{i, j=1}^{L} \prod_{i=1}^{L} \phi_{s, t}\left(x_{i}\right) d x_{i}\right) d s .
$$


Note that we throw out the $L=0$ term in the summation since it is always 1 and has $s$-derivative to be 0 . It turns out that we can interchange the order of derivative, integration and summation for the right hand side of the above display, for which we formulate as a lemma. The proof of it is deferred to Section 4.2.

Lemma 2.7. We have

$$
\begin{aligned}
& \int_{0}^{1} s^{-\alpha} \partial_{s}^{n}\left(\sum_{L=1}^{\infty} \frac{1}{L !} \int_{\mathbb{R}^{L}} \operatorname{Pf}\left[K\left(x_{i}, x_{j}\right)\right]_{i, j=1}^{L} \prod_{i=1}^{L} \phi_{s, t}\left(x_{i}\right) d x_{i}\right) d s \\
& =\sum_{L=1}^{\infty} \frac{1}{L !} \int_{0}^{1} s^{-\alpha} d s \int_{\mathbb{R}^{L}} \operatorname{Pf}\left[K\left(x_{i}, x_{j}\right)\right]_{i, j=1}^{L} \partial_{s}^{n}\left(\prod_{i=1}^{L} \phi_{s, t}\left(x_{i}\right)\right) d x_{1} \ldots d x_{L} .
\end{aligned}
$$

Consequently, it follows from (2.8) and the above display that

$$
\int_{0}^{1} s^{-\alpha} \partial_{s}^{n} \mathbb{E}\left[\exp \left(-s \mathcal{Z}^{h f}(2 t, 0) e^{\frac{t}{12}}\right)\right] d s=\sum_{L=1}^{\infty} \frac{1}{L !} \int_{0}^{1} s^{-\alpha} d s \int_{\mathbb{R}^{L}} \operatorname{Pf}\left[K\left(x_{i}, x_{j}\right)\right]_{i, j=1}^{L} \partial_{s}^{n}\left(\prod_{i=1}^{L} \phi_{s, t}\left(x_{i}\right)\right) d x_{1} \ldots d x_{L} .
$$

We set the first term in the right hand side summation of (2.10) as $\mathcal{A}_{p}(t)$ and the higher order terms as $\mathcal{B}_{p, L}(t)(L \geq 2)$, i.e.

$$
\begin{aligned}
\mathcal{A}_{p}(t) & =\frac{(-1)^{n}}{\Gamma(1-\alpha)} \int_{0}^{1} s^{-\alpha} \int_{\mathbb{R}} K_{12}(x, x)\left(\partial_{s}^{n} \phi_{s, t}(x)\right) d x \\
\mathcal{B}_{p, L}(t) & =\frac{(-1)^{n}}{\Gamma(1-\alpha) L !} \int_{0}^{1} s^{-\alpha} \int_{\mathbb{R}^{L}} \operatorname{Pf}\left[K\left(x_{i}, x_{j}\right)\right]_{i, j=1}^{L} \partial_{s}^{n}\left(\prod_{i=1}^{L} \phi_{s, t}\left(x_{i}\right)\right) d x_{1} \ldots d x_{L}, \quad L \geq 2 .
\end{aligned}
$$

Under this notation, the left hand side of (2.10) equals $\mathcal{A}_{p}(t)+\sum_{p=2}^{\infty} \mathcal{B}_{p, L}(t)$. Referring to (2.7), we obtain

$$
\mathbb{E}\left[\mathcal{Z}^{\mathrm{hf}}(2 t, 0)^{p} e^{\frac{p t}{12}}\right]=\mathcal{A}_{p}(t)+\sum_{L=2}^{\infty} \mathcal{B}_{p, L}(t)+\mathcal{R}_{p}(t) .
$$

We want to show that the logarithm of the left hand side in the above display, after divided by $t$ and letting $t \rightarrow \infty$, converges to $p^{3} / 3$. By Lemma 2.6, $\left|\mathcal{R}_{p}(t)\right|$ is uniformly upper bounded by a constant for all $t>0$. Therefore, to prove Theorem 1.1, it suffices to demonstrate that the following facts for $\mathcal{A}_{p}(t)$ and $\sum_{L=2}^{\infty}\left|\mathcal{B}_{p, L}(t)\right|$.

Proposition 2.8. For fixed $p \in \mathbb{R}_{>0}, \lim _{t \rightarrow \infty} \frac{1}{t} \log \mathcal{A}_{p}(t)=e^{\frac{p^{3}}{3}}$.

Proposition 2.9. For fixed $p \in \mathbb{R}_{>0}$, $\lim \sup _{t \rightarrow \infty} \frac{1}{t} \log \left(\sum_{L=2}^{\infty}\left|\mathcal{B}_{p, L}(t)\right|\right) \leq e^{\frac{p^{3}}{3}-\delta_{p}}$ with $\delta_{p}=\min \left(\frac{2}{3}, \frac{p^{3}}{4}\right)$.

We will prove the two propositions in Section 3 and Section 5. Let us first conclude the proof of Theorem 1.1.

Proof of Theorem 1.1. For part (i), from Proposition 2.8 and 2.9, we know that $\mathcal{A}_{p}(t)$ grows exponentially faster than $\sum_{L=2}^{\infty}\left|\mathcal{B}_{p, L}(t)\right|$ as $t \rightarrow \infty$. Along with the fact that $\left|\mathcal{R}_{p}(t)\right|$ is upper bounded by a constant for all $t$, there exists $t_{0}>0$ such that for all $t>t_{0}$,

$$
\sum_{L=2}^{\infty}\left|\mathcal{B}_{p, L}(t)\right|+\left|\mathcal{R}_{p}(t)\right| \leq \frac{1}{2} \mathcal{A}_{p}(t)
$$

Referring to the decomposition (2.13) and using triangle inequality, we see that for $t>t_{0}$,

$$
\log \left(\frac{1}{2} \mathcal{A}_{p}(t)\right) \leq \log \mathbb{E}\left[\mathcal{Z}^{\mathrm{hf}}(2 t, 0)^{p}\right] \leq \log \left(\frac{3}{2} \mathcal{A}_{p}(t)\right) .
$$

Dividing very term in the above display by $t$, Theorem 1.1 (i) follows easily from Proposition 2.8 as we take $t \rightarrow \infty$. Since we know that $t^{-1} \lim _{t \rightarrow \infty} \log \mathbb{E}\left[\mathcal{Z}^{\mathrm{hf}}(2 t, 0)^{p} \exp \left(\frac{p t}{12}\right)\right]=p^{3} / 3$. Applying [GL20, Proposition 1.12] with $h(p)=\frac{p^{3}}{3}$, we obtain the upper tail LDP with rate function to be $-\sup _{p>0}\left(p s-p^{3} / 3\right)=\frac{2}{3} s^{\frac{3}{2}}$, thus we obtain Theorem 1.1 (ii). 


\section{Asymptotic of $\mathcal{A}_{p}(t)$ : Proof of Proposition 2.8}

In this section, we prove Proposition 2.8. One crucial step is Lemma 3.2, whose proof relies on Lemma B.1 and a steepest descent type analysis. Throughout the rest of the paper, we use $C, C_{1}, C_{2}$ to denote a constant, which may vary from line to line. We might not generally specify when irrelevant terms are being absorbed into the constants. We might also write $C(a), C(a, b)$ when we want to specify which parameters the constant depends on.

Lemma 3.1. Denote $\mathrm{B}(u, v)$ to be the beta function $\int_{0}^{1} x^{u-1}(1-x)^{v-1} d x$. For $\gamma>0, \alpha<1$ and $\alpha+\beta>1$,

$$
\int_{0}^{\infty} \frac{s^{-\alpha}}{(1+\gamma s)^{\beta}} d s=\gamma^{\alpha-1} \mathrm{~B}(1-\alpha, \beta+\alpha-1)
$$

Proof. Via a change of variable $s=\frac{t}{\gamma(1-t)}$, we get

$$
\int_{0}^{\infty} \frac{s^{-\alpha} d s}{(1+\gamma s)^{\beta}}=\gamma^{\alpha-1} \int_{0}^{1} t^{-\alpha}(1-t)^{\alpha+\beta-2} d t=\gamma^{\alpha-1} \mathrm{~B}(1-\alpha, \beta+\alpha-1) .
$$

Lemma 3.2. For fixed $p, t_{0}>0$, there exists constant $C=C\left(p, t_{0}\right)$ such that for all $t>t_{0}$,

$$
\frac{1}{C} t^{-\frac{2}{3}} e^{\frac{1}{3} p^{3} t} \leq \int_{0}^{\infty} K_{12}\left(t^{\frac{2}{3}} x, t^{\frac{2}{3}} x\right) e^{p t x} d x \leq C t^{-\frac{2}{3}} e^{\frac{1}{3} p^{3} t}
$$

Proof. Throughout the proof we write $C=C\left(p, t_{0}\right)$ and denote by $U_{p}(x)=-\frac{2}{3} x^{\frac{3}{2}}+p x$. Using the inequality in Lemma B.1 (i),

$$
\frac{1}{C} \int_{0}^{\infty} \frac{e^{t U_{p}(x)}}{\left(1+t^{\frac{2}{3}} x\right)^{\frac{1}{4}}} d x \leq \int_{0}^{\infty} K_{12}\left(t^{\frac{2}{3}} x, t^{\frac{2}{3}} x\right) e^{p t x} d x \leq C \int_{0}^{\infty} \frac{e^{t U_{p}(x)}}{\left(1+t^{\frac{2}{3}} x\right)^{\frac{1}{4}}} d x
$$

The proof is completed if we can show there exists a constant $C$ such that for all $t>t_{0}$,

$$
\frac{1}{C} t^{-\frac{2}{3}} e^{\frac{p^{3} t}{3}} \leq \int_{0}^{\infty} \frac{e^{t U_{p}(x)}}{\left(1+t^{\frac{2}{3}} x\right)^{\frac{1}{4}}} d x \leq C t^{-\frac{2}{3}} e^{\frac{p^{3} t}{3}}
$$

An elementary calculus tells that the maximum of $U_{p}(x)=-\frac{2}{3} x^{\frac{3}{2}}+p x$ on $[0, \infty)$ is reached at $x=p$, with $U_{p}(p)=\frac{1}{3} p^{3}$. So it is natural to expect that the main contribution of the integral in the above display comes around a small region around $x=p$. Having this intuition in mind, we let $\mathbf{q}=\frac{p}{4}$ decompose

$$
\int_{0}^{\infty} \frac{e^{t U_{p}(x)}}{\left(1+t^{\frac{2}{3}} x\right)^{\frac{1}{4}}} d x=\left(\int_{\left[(p-\mathrm{q})^{2},(p+\mathrm{q})^{2}\right]}+\int_{\mathbb{R}_{>0} \backslash\left[(p-\mathrm{q})^{2},(p+\mathrm{q})^{2}\right]}\right) \frac{e^{t U_{p}(x)}}{\left(1+t^{\frac{2}{3}} x\right)^{\frac{1}{4}}} d x=\mathcal{K}_{1}+\mathcal{K}_{2} .
$$

It suffices to analyze $\mathcal{K}_{1}$ and $\mathcal{K}_{2}$ respectively. For $\mathcal{K}_{1}$, we make a change of variable $x=(p+r)^{2}$. Noting that $U_{p}\left((p+r)^{2}\right)=\frac{p^{3}}{3}-\left(\frac{2}{3} r+p\right) r^{2}$, we get

$$
\mathcal{K}_{1}=\int_{-\mathrm{q}}^{\mathrm{q}} \frac{2(p+r) e^{t U_{p}\left((p+r)^{2}\right)}}{\left(1+t^{\frac{2}{3}}(p+r)^{2}\right)^{\frac{1}{4}}} d r=e^{\frac{p^{3} t}{3}} \int_{-\mathrm{q}}^{\mathrm{q}} \frac{2(p+r) e^{-t\left(\frac{2}{3} r+p\right) r^{2}}}{\left(1+t^{\frac{2}{3}}(p+r)^{2}\right)^{\frac{1}{4}}} d r
$$

Recall that $\mathrm{q}=\frac{p}{4}$, so there exists a constant $C=C\left(p, t_{0}\right)$ such that for all $r \in\left[-\frac{p}{4}, \frac{p}{4}\right]$ and $t>t_{0}$,

$$
\frac{e^{-C t r^{2}}}{C t^{\frac{1}{6}}} \leq \frac{2(p+r) e^{-t\left(\frac{2}{3} r+p\right) r^{2}}}{\left(1+t^{\frac{2}{3}}(p+r)^{2}\right)^{\frac{1}{4}}} \leq \frac{C e^{-\frac{1}{C} t r^{2}}}{t^{\frac{1}{6}}} .
$$

By a change of variable $r \rightarrow t^{-\frac{1}{2}} r$, there exists constant $C_{1}$ such that for $t>t_{0}$

$$
C_{1}^{-1} t^{-\frac{2}{3}} \leq \int_{-\mathrm{q}}^{\mathrm{q}} \frac{e^{-C t r^{2}}}{C t^{\frac{1}{6}}} \leq \int_{-\mathrm{q}}^{\mathrm{q}} \frac{C e^{-\frac{1}{C} t r^{2}}}{t^{\frac{1}{6}}} \leq C_{1} t^{-\frac{2}{3}}
$$

Integrating the terms in (3.4) from $-\mathrm{q}$ to $\mathrm{q}$ and utilizing the displayed inequality above and (3.3), we conclude that $\frac{1}{C} t^{-\frac{2}{3}} e^{\frac{p^{3} t}{3}} \leq \mathcal{K}_{1} \leq C t^{-\frac{2}{3}} e^{\frac{p^{3} t}{3}}$ for $t>t_{0}$.

For $\mathcal{K}_{2}$, by a change of variable $x=r^{2}$ and noting $U_{p}\left(r^{2}\right)=\frac{p^{3}}{3}-(r-p)^{2}\left(\frac{2}{3} r+\frac{1}{3} p\right)$, we have

$$
\mathcal{K}_{2}=e^{\frac{p^{3} t}{3}} \int_{\mathbb{R}_{>0} \backslash[p-\mathbf{q}, p+\mathbf{q}]} \frac{e^{t(r-p)^{2}\left(-\frac{2}{3} r-\frac{1}{3} p\right)}}{\left(1+t^{\frac{2}{3}} r^{2}\right)^{\frac{1}{4}}} d r \leq e^{\frac{t\left(p^{3}-p \mathbf{q}^{2}\right)}{3}} \int_{\mathbb{R}_{>0} \backslash[p-\mathbf{q}, p+\mathbf{q}]} \frac{e^{-\frac{2}{3} t r(r-p)^{2}}}{\left(1+t^{\frac{2}{3}} r^{2}\right)^{\frac{1}{4}}} d r
$$


The inequality in the above display follows from noticing $\frac{1}{3}(r-p)^{2} p \geq \frac{p \mathrm{q}^{2}}{3}$ when $r \notin[p-\mathrm{q}, p+\mathrm{q}]$. For the integral on the right hand side of the above display, we find that $\int_{\mathbb{R}_{>0} \backslash[p-\mathbf{q}, p+\mathbf{q}]} \frac{e^{-\frac{2}{3} t r(r-p)^{2}}}{\left(1+t^{\frac{2}{3}} r^{2}\right)^{\frac{1}{4}}} d r \leq \int_{\mathbb{R}_{>0}} e^{-\frac{2}{3} t \mathbf{q}^{2} r} d r=\frac{3}{2 \mathbf{q}^{2} t}$. Since we assume $t \geq t_{0}$, by taking $C=\frac{3}{2 \mathrm{q}^{2} t_{0}}$, we know that

$$
0 \leq \mathcal{K}_{2} \leq \frac{3}{2 q^{2} t} e^{\frac{t\left(p^{3}-p q^{2}\right)}{3}} \leq C e^{\frac{t\left(p^{3}-p q^{2}\right)}{3}}
$$

Combining this with (3.3) and recall from (3.2) that $\int_{0}^{\infty} \frac{e^{t U_{p}(x)}}{\left(1+t^{\frac{2}{3}} x\right)^{\frac{1}{4}}} d x=\mathcal{K}_{1}+\mathcal{K}_{2}$, we see that $\mathcal{K}_{1}$ is the dominating term. This completes the proof of (3.1).

We are now ready to prove Proposition 2.8 .

Proof of Proposition 2.8. Recall from (2.5) that $\phi_{s, t}(x)=\frac{1}{\sqrt{1+4 s \exp \left(t^{1 / 3} x\right)}}-1$, so

$$
\partial_{s}^{n} \phi_{s, t}(x)=(-2)^{n}(2 n-1) ! !\left(1+4 s \exp \left(t^{\frac{1}{3}} x\right)\right)^{-\frac{2 n+1}{2}} .
$$

By Fubini's theorem, we switch the order of integration on the right hand side of $(2.11)$, hence

$$
\mathcal{A}_{p}(t)=\frac{2^{n}(2 n-1) ! !}{\Gamma(1-\alpha)} \int_{\mathbb{R}} K_{12}(x, x) e^{n t^{\frac{1}{3}} x} d x \int_{0}^{1} \frac{s^{-\alpha}}{\left(1+4 s \exp \left(t^{\frac{1}{3}} x\right)\right)^{\frac{2 n+1}{2}}} d s
$$

Writing the integral w.r.t $s$ in the above display as $\int_{0}^{1}=\int_{0}^{\infty}-\int_{1}^{\infty}$, we get $\mathcal{A}_{p}(t)=\mathcal{A}_{p}^{\prime}(t)-\mathcal{A}_{p}^{\prime \prime}(t)$, where

$$
\begin{aligned}
& \mathcal{A}_{p}^{\prime}(t)=\frac{2^{n}(2 n-1) ! !}{\Gamma(1-\alpha)} \int_{\mathbb{R}} K_{12}(x, x) e^{n t^{\frac{1}{3}}} x d x \int_{0}^{\infty} \frac{s^{-\alpha}}{\left(1+4 s \exp \left(t^{\frac{1}{3}} x\right)\right)^{\frac{2 n+1}{2}}} d s, \\
& \mathcal{A}_{p}^{\prime \prime}(t)=\frac{2^{n}(2 n-1) ! !}{\Gamma(1-\alpha)} \int_{\mathbb{R}} K_{12}(x, x) e^{n t^{\frac{1}{3}} x} d x \int_{1}^{\infty} \frac{s^{-\alpha}}{\left(1+4 s \exp \left(t^{\frac{1}{3}} x\right)\right)^{\frac{2 n+1}{2}}} d s .
\end{aligned}
$$

To conclude our proof of Proposition 2.8, it suffices to show the following propositions.

Proposition 3.3. For fixed $p, t_{0}>0$ there exists $C=C\left(p, t_{0}\right)$ such that for all $t>t_{0}, \frac{1}{C} e^{\frac{1}{3} p^{3} t} \leq \mathcal{A}_{p}^{\prime}(t) \leq C e^{\frac{1}{3} p^{3} t}$.

Proposition 3.4. For fixed $p, t_{0}>0$, there exists $C=C\left(p, t_{0}\right)$ such that for all $t>t_{0},\left|\mathcal{A}_{p}^{\prime \prime}(t)\right| \leq C$.

Let us first complete our proof of Proposition 2.8 using Proposition 3.3 and 3.4. Recall $\mathcal{A}_{p}(t)=\mathcal{A}_{p}^{\prime}(t)-\mathcal{A}_{p}^{\prime \prime}(t)$. With the help of these lemmas, it is clear that $\mathcal{A}_{p}^{\prime}(t)$ is the dominating term for large enough $t$. Hence,

$$
\lim _{t \rightarrow \infty} \frac{1}{t} \log \mathcal{A}_{p}(t)=\lim _{t \rightarrow \infty} t^{-1} \log \mathcal{A}_{p}^{\prime}(t)=\frac{p^{3}}{3} .
$$

This completes our proof of Proposition 2.8.

For the rest of this section, we prove Proposition 3.3 and 3.4 respectively.

Proof of Proposition 3.3. Applying Lemma 3.1 to the second integral on the right hand side of (3.6) (with $\beta=\frac{2 n+1}{2}$ and $\gamma=4 \exp \left(t^{\frac{1}{3}} x\right)$ ), we see that (recall $p=n-1+\alpha$ )

$$
\mathcal{A}_{p}^{\prime}(t)=c_{p} \int_{-\infty}^{\infty} K_{12}(x, x) \exp \left(p t^{\frac{1}{3}} x\right) d x .
$$

where $c_{p}$ is a constant that equals $\frac{2^{n}(2 n-1) ! ! 4^{\alpha-1}}{\Gamma(1-\alpha)} \mathrm{B}\left(1-\alpha, \frac{2 n-1}{2}+\alpha\right)$. We will not use this explicit expression of $c_{p}$ and later we just write it as a generic constant $C$. By a change of variable $x \rightarrow t^{\frac{2}{3}} x$, we have $\mathcal{A}_{p}^{\prime}(t)=$ $C t^{\frac{2}{3}} \int_{-\infty}^{\infty} K_{12}\left(t^{2 / 3} x, t^{2 / 3} x\right) \exp (p t x) d x$. Decompose the integral region into $(-\infty, 0) \cup[0, \infty)$, we obtain

$$
\mathcal{A}_{p}^{\prime}(t)=C t^{\frac{2}{3}}\left(\int_{0}^{\infty} K_{12}\left(t^{\frac{2}{3}} x, t^{\frac{2}{3}} x\right) e^{p t x} d x+\int_{-\infty}^{0} K_{12}\left(t^{\frac{2}{3}} x, t^{\frac{2}{3}} x\right) e^{p t x} d x\right)
$$

For the first integral on the right hand side of the (3.8), referring to Lemma 3.2, we have

$$
\frac{1}{C_{1}} t^{-\frac{2}{3}} e^{\frac{p^{3} t}{3}} \leq \int_{0}^{\infty} K_{12}\left(t^{\frac{2}{3}} x, t^{\frac{2}{3}} x\right) e^{p t x} d x \leq C_{1} t^{-\frac{2}{3}} e^{\frac{p^{3} t}{3}}
$$


For the second integral on the right hand side of (3.8), we apply Lemma B.1 (ii) and get $t>t_{0}$

$$
0 \leq \int_{-\infty}^{0} K_{12}\left(t^{\frac{2}{3}} x, t^{\frac{2}{3}} x\right) e^{p t x} d x \leq C_{2} \int_{-\infty}^{0}\left(1-t^{\frac{2}{3}} x\right)^{\frac{1}{2}} e^{p t x} d x \leq C_{3}
$$

where $C_{1}, C_{2}, C_{3}$ only depends on $p, t_{0}$. Combining (3.8), (3.9) and (3.10), we know that $C C_{1}^{-1} e^{\frac{p^{3} t}{3}} \leq \mathcal{A}_{p}^{\prime}(t) \leq$ $C C_{1} e^{\frac{p^{3} t}{3}}+C C_{3} t^{\frac{2}{3}}$. Note that $t^{\frac{2}{3}}$ can be upper bounded by a constant times $e^{\frac{p^{3}}{3} t}$ when $t>t_{0}$, we conclude Proposition 3.3.

Proof of Proposition 3.4. Recall the expression of $\mathcal{A}_{p}^{\prime \prime}(t)$ from (3.7). Since $K_{12}(x, x)$ is non-negative for all $x$, $\mathcal{A}_{p}^{\prime \prime}(t)$ is lower bounded by 0 . To get the upper bound, we decompose $\mathcal{A}_{p}^{\prime \prime}(t)=\frac{2^{n}(2 n-1) ! !}{\Gamma(1-\alpha)}\left(\mathrm{A}_{1}+\mathrm{A}_{2}\right)$ where

$$
\begin{aligned}
& \mathrm{A}_{1}=\int_{0}^{\infty} K_{12}(x, x) \exp \left(n t^{\frac{1}{3}} x\right) d x \int_{1}^{\infty} \frac{s^{-\alpha}}{\left(1+4 s \exp \left(t^{\frac{1}{3}} x\right)\right)^{\frac{2 n+1}{2}}} d s \\
& \mathrm{~A}_{2}=\int_{-\infty}^{0} K_{12}(x, x) \exp \left(n t^{\frac{1}{3}} x\right) d x \int_{1}^{\infty} \frac{s^{-\alpha}}{\left(1+4 s \exp \left(t^{\frac{1}{3}} x\right)\right)^{\frac{2 n+1}{2}}} d s
\end{aligned}
$$

Let us upper bound $\mathrm{A}_{1}$ and $\mathrm{A}_{2}$ respectively. We start with $\mathrm{A}_{1}$, using $1+4 s \exp \left(t^{\frac{1}{3}} x\right) \geq 4 s \exp \left(t^{\frac{1}{3}} x\right)$,

$$
\int_{1}^{\infty} \frac{s^{-\alpha}}{\left(1+4 s \exp \left(t^{\frac{1}{3}} x\right)\right)^{\frac{2 n+1}{2}}} \leq \int_{1}^{\infty} s^{-\alpha}\left(4 s \exp \left(t^{\frac{1}{3}} x\right)\right)^{-\frac{2 n+1}{2}} d s=\frac{\exp \left(-\frac{2 n+1}{2} t^{\frac{1}{3}} x\right)}{2^{2 n+1}\left(\frac{2 n-1}{2}+\alpha\right)} .
$$

Applying this inequality to the right hand side of (3.11), we have $\mathrm{A}_{1} \leq C \int_{0}^{\infty} K_{12}(x, x) \exp \left(-\frac{1}{2} t^{\frac{1}{3}} x\right) d x$. Using Lemma B.1 (i), for all $t>0$, there exists a constant $C_{1}$ such that

$$
\mathrm{A}_{1} \leq C \int_{0}^{\infty} \frac{e^{-\frac{2}{3} x^{\frac{3}{2}}}}{(1+x)^{\frac{1}{4}}} e^{-\frac{1}{2} t^{\frac{1}{3}} x} d x \leq C \int_{0}^{\infty} \frac{e^{-\frac{2}{3} x^{\frac{3}{2}}}}{(1+x)^{\frac{1}{4}}} d x=C_{1}
$$

We continue to upper bound $A_{2}$. Relaxing the integral region from $[1, \infty)$ to $[0, \infty)$, we get

$$
\int_{1}^{\infty} \frac{s^{-\alpha}}{\left(1+4 s \exp \left(t^{\frac{1}{3}} x\right)\right)^{\frac{2 n+1}{2}}} \leq \int_{0}^{\infty} \frac{s^{-\alpha}}{\left(1+4 s \exp \left(t^{\frac{1}{3}} x\right)\right)^{\frac{2 n+1}{2}}} d s=4^{\alpha-1} \mathrm{~B}\left(1-\alpha, \frac{2 n-1+2 \alpha}{2}\right) e^{(\alpha-1) t^{\frac{1}{3}} x} .
$$

The equality above follows from a change of variable $s \rightarrow \frac{1}{4} \exp \left(-t^{\frac{1}{3}} x\right) s$ and Lemma 3.1. Due to the above display (set the product of $4^{\alpha-1}$ and the beta function to be a constant $C$ )

$$
\mathrm{A}_{2} \leq C \int_{-\infty}^{0} K_{12}(x, x) \exp \left((n+\alpha-1) t^{\frac{1}{3}} x\right) d x=C \int_{-\infty}^{0} K_{12}(x, x) \exp \left(p t^{\frac{1}{3}} x\right) d x .
$$

Using Lemma B.1 to upper bound $K_{12}(x, x)$ for negative $x$, there exists a constant $C_{2}$ such that for all $t>t_{0}$,

$$
\mathrm{A}_{2} \leq C \int_{-\infty}^{0} \sqrt{1-x} e^{p t^{\frac{1}{3}} x} d x=C \int_{-\infty}^{0} \sqrt{1-x} e^{p t_{0}^{\frac{1}{3}} x} d x=C_{2}
$$

Having $\mathrm{A}_{1}, \mathrm{~A}_{2}$ upper bounded by a constant uniformly for $t>t_{0}$, we conclude our lemma by recalling that $\mathcal{A}_{p}^{\prime \prime}(t)$ is a constant multiple of $A_{1}+A_{2}$.

\section{Controlling the Pfaffian and Proof of Lemma 2.7}

In this section, we give two upper bounds of the $L$-th $\operatorname{Pfaffian} \operatorname{Pf}\left[K\left(x_{i}, x_{j}\right)\right]_{i, j=1}^{L}$ uniformly for all $L$. This is the main technical contribution of our paper. The purpose is two folded. First, these upper bounds are the crucial inputs to the proof of Proposition 2.9 presented in the next section. Secondly, they can be used to validate the interchange of derivative, integration and summation in Lemma 2.7. 
4.1. Controlling the Pfaffian. We obtain two upper bounds for $\operatorname{Pf}\left[K\left(x_{i}, x_{j}\right)\right]_{i, j=1}^{L}$ for all $L$. Each upper bound has its advantage. The first upper bound has slower growth in $L$ and slower exponential decay in $x_{i}$ as $x_{i} \rightarrow \infty$. The second upper bound has faster exponential decay in $x_{i}$ but also a more rapid growth in $L$. For the proof of Proposition 2.9, we will use both of the upper bounds to control various terms in $\mathcal{B}_{p, L}$ depending on how large the $L$ is. To prove these bounds, we utilize various bounds for $K_{i j}(x, y), i, j \in\{1,2\}$ that are established in Lemma B.2.

Define $F_{\alpha, \beta}(x)=e^{-\alpha x^{\frac{3}{2}}} \mathbf{1}_{\{x \geq 0\}}+(1-x)^{\beta} \mathbf{1}_{\{x<0\}}$. It is clear that $F_{\alpha_{1}, \beta_{1}}(x) F_{\alpha_{2}, \beta_{2}}(x)=F_{\alpha_{1}+\alpha_{2}, \beta_{1}+\beta_{2}}(x)$. In addition, for $\beta_{1} \leq \beta_{2}$, we have $F_{\alpha, \beta_{1}}(x) \leq F_{\alpha, \beta_{2}}(x)$.

Proposition 4.1. There exists constant $C$ such that for all $L \in \mathbb{Z}_{\geq 1}$,

(i) $\left|\operatorname{Pf}\left[K\left(x_{i}, x_{j}\right)\right]_{i, j=1}^{L}\right| \leq(2 L)^{L / 2} C^{L} \prod_{i=1}^{L} F_{\frac{1}{3}, 2}\left(x_{i}\right)$

(ii) $\left|\operatorname{Pf}\left[K\left(x_{i}, x_{j}\right)\right]_{i, j=1}^{L}\right| \leq \sqrt{(2 L) !} C^{L} \prod_{i=1}^{L} F_{\frac{2}{3}, 2}\left(x_{i}\right)$

Looking at the growth of these upper bounds in terms of $L$, by Stirling's formula, $\sqrt{(2 L) !} \sim e^{-L}(2 L)^{L+\frac{1}{4}}(2 \pi)^{\frac{1}{4}}$ which grows faster than $(2 L)^{\frac{L}{2}}$. On the other hand, in terms of $x$, as $x_{i} \rightarrow \infty$, the second upper bound decays with speed $\exp \left(-\frac{2}{3} x_{i}^{\frac{3}{2}}\right)$, which is faster than that the first upper bound whose exponent is $\exp \left(-\frac{1}{3} x_{i}^{\frac{3}{2}}\right)$.

Proof of Proposition 4.1 (i). The idea for proving Proposition 4.1 (i) is as follows. Up to a sign, the Pfaffian of a matrix equals the square root of its determinant. We apply Hadamard's inequality to upper bound the determinant in terms of the product of $\ell_{\infty}$-norms of each row of the matrix. Finally, we apply Lemma B.2 to control these $\ell_{\infty}$-norms.

Now we start our proof. It is well-known that

$$
\left|\operatorname{Pf}\left[K\left(x_{i}, x_{j}\right)\right]_{i, j=1}^{L}\right|=\sqrt{\operatorname{det}\left[K\left(x_{i}, x_{j}\right)\right]_{i, j=1}^{L}} .
$$

Notice that each entry $K\left(x_{i}, x_{j}\right)$ is a $2 \times 2$ matrix and $\left[K\left(x_{i}, x_{j}\right)\right]_{i, j=1}^{L}$ is a $2 L \times 2 L$ matrix. Denote $r_{i}$ to be the $i$-th row vector of this matrix. Applying Hadamard's inequality for the determinant on the right hand side above, we see that

$$
\left|\operatorname{Pf}\left[K\left(x_{i}, x_{j}\right)\right]_{i, j=1}^{L}\right| \leq(2 L)^{\frac{L}{2}} \sqrt{\prod_{i=1}^{2 L}\left\|\mathrm{r}_{i}\right\|_{\infty}}
$$

where $\|\cdot\|_{\infty}$ denotes the $\ell^{\infty}$-norm of a vector. It suffices to upper bound each $\left\|r_{i}\right\|_{\infty}$, we do that according to the parity of $i$. For each $k=1, \ldots, L$, the vector $r_{2 k-1}$ is composed of the elements $K_{11}\left(x_{k}, x_{j}\right)$ and $K_{12}\left(x_{k}, x_{j}\right)$ for $j=1, \ldots, L$, thus

$$
\left\|\mathrm{r}_{2 k-1}\right\|_{\infty}=\max _{j=1, \ldots, L}\left(\max \left(\left|K_{11}\left(x_{k}, x_{j}\right)\right|,\left|K_{12}\left(x_{k}, x_{j}\right)\right|\right)\right)
$$

Using the Lemma B.2 (a) and (b) for $K_{11}$ and $K_{12}$ respectively, we know that there exists constant $C$ such that $\left|K_{11}\left(x_{k}, x_{j}\right)\right| \leq C F_{\frac{2}{3}, \frac{5}{4}}\left(x_{k}\right)$ and $\left|K_{12}\left(x_{k}, x_{j}\right)\right| \leq C F_{\frac{2}{3}, \frac{3}{4}}\left(x_{k}\right)$. This implies that $\left\|\mathrm{r}_{2 k-1}\right\|_{\infty} \leq C F_{\frac{2}{3}, \frac{5}{4}}\left(x_{k}\right)$. Similarly, the row vector $r_{2 k}$ is composed of $K_{21}\left(x_{k}, x_{j}\right)$ and $K_{22}\left(x_{k}, x_{j}\right)$, using Lemma B.2 (b) and (c) for $K_{12}$ and $K_{22}$ respectively (note that $\left|K_{21}\left(x_{k}, x_{j}\right)\right|=\left|K_{12}\left(x_{j}, x_{k}\right)\right| \leq C F_{0, \frac{3}{4}}\left(x_{k}\right)$ ), we get

$$
\left\|\mathrm{r}_{2 k}\right\|_{\infty}=\max _{j=1, \ldots, L}\left(\max \left(\left|K_{21}\left(x_{k}, x_{j}\right)\right|,\left|K_{22}\left(x_{k}, x_{j}\right)\right|\right)\right) \leq C F_{0, \frac{3}{4}}\left(x_{k}\right)
$$

Inserting the upper bounds for $\left\|r_{2 k-1}\right\|$ and $\left\|r_{2 k}\right\|$ into the right hand side of (4.2), we have

$$
\left|\operatorname{Pf}\left[K\left(x_{i}, x_{j}\right)\right]_{i, j=1}^{L}\right| \leq(2 L)^{\frac{L}{2}} C^{L} \sqrt{\prod_{k=1}^{L} F_{\frac{2}{3}, \frac{5}{4}}\left(x_{k}\right)} \cdot \sqrt{\prod_{k=1}^{L} F_{0, \frac{3}{4}}\left(x_{k}\right)} \leq(2 L)^{\frac{L}{2}} C^{L} \prod_{k=1}^{L} F_{\frac{1}{3}, 2}\left(x_{k}\right) .
$$

The last equality follows from $\sqrt{F_{\frac{2}{3}, \frac{5}{4}}\left(x_{k}\right) F_{0, \frac{3}{4}}\left(x_{k}\right)}=F_{\frac{1}{3}, 1}\left(x_{k}\right) \leq F_{\frac{1}{3}, 2}\left(x_{k}\right)$. This completes our proof.

To prove Proposition 4.1 (ii), we upper bound the determinant in a different way. Instead of using Hadamard's inequality, we work with the permutation expansion of the determinant and seek to upper bound each term 
therein. We introduce some notations. Rewrite the $2 L \times 2 L$ matrix $\left[K\left(x_{i}, x_{j}\right)\right]_{i, j=1}^{L}$ as $[\mathrm{D}(i, j)]_{i, j=1}^{2 L}$ in a way that for all $i, j \in\{1, \ldots, L\}$,

$$
\begin{array}{ll}
\mathrm{D}(2 i-1,2 j-1)=K_{11}\left(x_{i}, x_{j}\right), & \mathrm{D}(2 i-1,2 j)=K_{12}\left(x_{i}, x_{j}\right), \\
\mathrm{D}(2 i, 2 j-1)=K_{21}\left(x_{i}, x_{j}\right), & \mathrm{D}(2 i, 2 j)=K_{22}\left(x_{i}, x_{j}\right) .
\end{array}
$$

Note that we suppress the dependence on $x$ in the notation of D. Define the maps $\theta:\{1, \ldots, 2 L\} \rightarrow\{1, \ldots, L\}$ and $\tau:\{1, \ldots, 2 L\} \rightarrow\{0,1\}$ such that $\theta(n)=\left\lfloor\frac{n}{2}\right\rfloor$ and $\tau(n)=n-2\lfloor n / 2\rfloor$. It is clear that

$$
(\theta, \tau):\{1, \ldots, 2 L\} \rightarrow\{1, \ldots, L\} \times\{0,1\}
$$

is a bijection.

Lemma 4.2. There exists a constant $C$ such that for arbitrary $L$ and $i, j \in\{1, \ldots, 2 L\}$,

$$
|\mathrm{D}(i, j)| \leq C F_{\frac{2}{3} \tau(i), \frac{3}{4}}\left(x_{\theta(i)}\right) F_{\frac{2}{3} \tau(j), \frac{3}{4}}\left(x_{\theta(j)}\right) .
$$

Proof. We divide our proof of the above inequality into four cases. Case 1: $i, j$ are both odd. Case 2: $i, j$ are both even. Case 3: $i$ is odd and $j$ is even. Case 4: $i$ is even and $j$ is odd.

Case 1. $i, j$ are both odd. Then $\tau(i)=\tau(j)=1$ and $\mathrm{D}(i, j)=K_{11}\left(x_{\theta(i)}, x_{\theta(j)}\right)$. By Lemma B.2 (a),

$$
|\mathrm{D}(i, j)| \leq C F_{\frac{2}{3}, \frac{3}{4}}\left(x_{\theta(i)}\right) F_{\frac{2}{3}, \frac{3}{4}}\left(x_{\theta(j)}\right)
$$

Case 2. $i, j$ are both even. Then $\tau(i)=\tau(j)=0$ and $\mathrm{D}(i, j)=K_{22}\left(x_{\theta(i)}, x_{\theta(j)}\right)$. By Lemma B.2 (c),

$$
|\mathrm{D}(i, j)| \leq C F_{0, \frac{3}{4}}\left(x_{\theta(i)}\right) \leq C F_{0, \frac{3}{4}}\left(x_{\theta(i)}\right) F_{0, \frac{3}{4}}\left(x_{\theta(j)}\right) .
$$

where the second inequality above follows from $F_{0, \frac{3}{4}}(x)=1+(1-x)^{\frac{3}{4}} \mathbf{1}_{\{x \leq 0\}} \geq 1$.

Case 3. $i$ is odd and $j$ is even. Then $\tau(i)=1, \tau(j)=0$ and $\mathrm{D}(i, j)=K_{12}\left(x_{\theta(i)}, x_{\theta(j)}\right)$. Using Lemma B.2 (b),

$$
|\mathrm{D}(i, j)| \leq C F_{\frac{2}{3}, \frac{3}{4}}\left(x_{\theta(i)}\right) \leq C F_{\frac{2}{3}, \frac{3}{4}}\left(x_{\theta(i)}\right) F_{0, \frac{3}{4}}\left(x_{\theta(j)}\right) .
$$

Case 4. $i$ is even and $j$ is odd. Then $\tau(i)=0, \tau(j)=1$ and $\mathrm{D}(i, j)=K_{21}\left(x_{\theta(i)}, x_{\theta(j)}\right)$. This follows from Case 3 and the fact $K_{21}(x, y)=-K_{12}(y, x)$.

Since our discussion covers all the cases, we conclude our proof of the lemma.

Proof of Proposition 4.1 (ii). Referring to (4.1) and permutation expansion of the determinant,

$$
\left(\operatorname{Pf}\left[K\left(x_{i}, x_{j}\right)\right]_{i, j=1}^{L}\right)^{2}=\operatorname{det}\left[\mathrm{D}\left(x_{i}, x_{j}\right)\right]_{i, j=1}^{2 L}=\sum_{\sigma \in S_{2 L}} \prod_{i=1}^{L} \mathrm{D}(i, \sigma(i))
$$

where $S_{2 L}$ is the permutation group of $\{1, \ldots, 2 L\}$. Applying Lemma 4.2 for any permutation $\sigma \in S_{2 L}$,

$$
\left|\prod_{i=1}^{2 L} \mathrm{D}(i, \sigma(i))\right| \leq C^{2 L} \prod_{i=1}^{2 L} F_{\frac{2}{3} \tau(i), \frac{3}{4}}\left(x_{\theta(i)}\right) F_{\frac{2}{3} \tau(\sigma(i)), \frac{3}{4}}\left(x_{\theta(\sigma(i))}\right)=C^{2 L} \prod_{i=1}^{2 L} F_{\frac{2}{3} \tau(i), \frac{3}{4}}\left(x_{\theta(i)}\right) \prod_{i=1}^{2 L} F_{\frac{2}{3} \tau(i), \frac{3}{4}}\left(x_{\theta(i)}\right)
$$

Using the bijectivity of $(\tau, \theta):\{1,2 \ldots, 2 L\} \rightarrow\{1, \ldots, L\} \times\{0,1\}$, we see that for arbitrary $\sigma \in S_{2 L}$,

$$
\prod_{i=1}^{2 L} F_{\frac{2}{3} \tau(\sigma(i)), \frac{3}{4}}\left(x_{\theta(\sigma(i))}\right)=\prod_{i=1}^{2 L} F_{\frac{2}{3} \tau(i), \frac{3}{4}}\left(x_{\theta(i)}\right)=\prod_{i=1}^{L} F_{\frac{2}{3}, \frac{3}{4}}\left(x_{i}\right) F_{0, \frac{3}{4}}\left(x_{i}\right)=\prod_{i=1}^{L} F_{\frac{2}{3}, \frac{3}{2}}\left(x_{i}\right)
$$

This implies that for all permutation $\sigma \in S_{2 L}$, the absolute value of $\prod_{i=1}^{2 L} \mathrm{D}(i, \sigma(i))$ can be upper bounded by $C^{2 L}\left(\prod_{i=1}^{L} F_{\frac{2}{3}, \frac{3}{2}}\left(x_{i}\right)\right)^{2}$. Referring to (4.3), since there are $(2 L)$ ! terms in the summation on the right hand side,

$$
\left(\operatorname{Pf}\left[K\left(x_{i}, x_{j}\right)\right]_{i, j=1}^{L}\right)^{2} \leq(2 L) ! \max _{\sigma \in S_{2 L}}\left(\prod_{i=1}^{2 L} \mathrm{D}(i, \sigma(i))\right) \leq(2 L) ! C^{2 L}\left(\prod_{i=1}^{L} F_{\frac{2}{3}, \frac{3}{2}}\left(x_{i}\right)\right)^{2}
$$

Taking the square root for both sides above and using $F_{\frac{2}{3}, \frac{3}{2}}\left(x_{i}\right) \leq F_{\frac{2}{3}, 2}\left(x_{i}\right)$, this completes the proof. 
4.2. Proof of Lemma 2.7. In this subsection, we devote to justify that we can interchange the order of derivative, integration and summation for the right hand side (2.8) and provide a proof of Lemma 2.7. For the ensuing discussion, we denote $\partial_{s}^{k} \phi_{s, t}(x)$ by $\phi_{s, t}^{(k)}(x)$. In particular, when $k=0, \phi_{s, t}^{(k)}(x)$ coincides with $\phi_{s, t}(x)$.

Lemma 4.3. Fix $k$. Recall that $\phi_{s, t}(x)=\frac{1}{\sqrt{1+4 s \exp \left(t^{1 / 3} x\right)}}-1$, there exists $C=C(k)$ such that for all $s \geq 0$,

$$
\left|\phi_{s, t}^{(k)}(x)\right| \leq 1 \wedge 2 s e^{t^{\frac{1}{3}} x} \quad \text { if } k=0 ; \quad\left|\phi_{s, t}^{(k)}(x)\right| \leq C\left(e^{k t^{\frac{1}{3}} x} \wedge s^{-k}\right) \quad \text { if } k \in \mathbb{Z}_{\geq 1}
$$

Proof. It is easily verified that for all $y \geq 0,1-\frac{1}{\sqrt{1+y}} \leq 1 \wedge \frac{1}{2} y$. Taking $y=4 s \exp \left(t^{\frac{1}{3}} x\right)$ implies the first inequality. For the second inequality, we compute $\phi_{s, t}^{(k)}(x)=(-2)^{k}(2 k-1) ! ! \frac{\exp \left(k t^{\frac{1}{3}} x\right)}{\left(1+4 s \exp \left(t^{\frac{1}{3}} x\right)\right)^{\frac{2 k+1}{2}}}$. Lower bounding $\left(1+4 s \exp \left(t^{\frac{1}{3}} x\right)\right)^{\frac{2 k+1}{2}}$ by 1 , we get $\left|\phi_{s, t}^{(k)}(x)\right| \leq 2^{k}(2 k-1) ! ! \exp \left(k t^{\frac{1}{3}} x\right)$. On the other hand, we have

$$
\left|\phi_{s, t}^{(k)}(x)\right| \leq 2^{k}(2 k-1) ! ! \frac{\exp \left(k t^{\frac{1}{3} x}\right)}{\left(1+4 s \exp \left(t^{\frac{1}{3}} x\right)\right)^{k}} \leq s^{-k} 2^{k}(2 k-1) ! !
$$

This completes our proof.

Let us introduce a few notations. Define $\mathfrak{M}(L, n)=\left\{\vec{m}=\left(m_{1}, \ldots, m_{L}\right) \in \mathbb{Z}_{\geq 0}^{L}, \sum_{i=1}^{L} m_{i}=n\right\}$ and for $\vec{m} \in \mathfrak{M}(L, n)$, we set $\left(\begin{array}{c}n \\ \vec{m}\end{array}\right)=\frac{n !}{\prod_{i=1}^{L} m_{i} !}$.

Lemma 4.4. Fix $n \in \mathbb{Z}_{\geq 0}$ and $t>0$, there exists a constant $C=C(n, t)$ such that for all $L \in \mathbb{Z}_{\geq 1}$ and $s \in[0,1]$,

$$
\left|\int_{\mathbb{R}^{L}} \operatorname{Pf}\left[K\left(x_{i}, x_{j}\right)\right]_{i, j=1}^{L} \partial_{s}^{n}\left(\prod_{i=1}^{L} \phi_{s, t}\left(x_{i}\right)\right) d x_{1} \ldots d x_{L}\right| \leq(2 L)^{\frac{L}{2}} C^{L} .
$$

Proof. Throughout the proof, we write $C=C(n, t)$. By Leibniz's rule,

$$
\partial_{s}^{n}\left(\prod_{i=1}^{L} \phi_{s, t}\left(x_{i}\right)\right)=\sum_{\vec{m} \in \mathfrak{M}(L, n)}\left(\begin{array}{c}
n \\
\vec{m}
\end{array}\right) \prod_{i=1}^{L} \phi_{s, t}^{\left(m_{i}\right)}\left(x_{i}\right) .
$$

According to Lemma 4.3, there exists constant $C_{1}$ such that for each $0 \leq m_{i} \leq n$ and $s \in[0,1]$

$$
\left|\phi_{s, t}^{\left(m_{i}\right)}\left(x_{i}\right)\right| \leq 2 s e^{t^{\frac{1}{3}} x} \leq 2 e^{t^{\frac{1}{3}} x} \quad \text { if } m_{i}=0 ; \quad\left|\phi_{s, t}^{\left(m_{i}\right)}\left(x_{i}\right)\right| \leq C_{1} e^{m_{i} t^{\frac{1}{3}} x} \quad \text { if } 1 \leq m_{i} \leq n .
$$

Hence, we have $\left|\phi_{s, t}^{\left(m_{i}\right)}\left(x_{i}\right)\right| \leq C_{1}\left(\exp \left(t^{\frac{1}{3}} x\right) \vee \exp \left(t^{\frac{1}{3}} n x\right)\right)$. Taking the absolute value for both sides of (4.4) and applying triangle inequality,

$$
\left|\partial_{s}^{n}\left(\prod_{i=1}^{L} \phi_{s, T}\left(x_{i}\right)\right)\right| \leq C_{1}^{L} \sum_{\vec{m} \in \mathfrak{M}(L, n)}\left(\begin{array}{c}
n \\
\vec{m}
\end{array}\right) \prod_{i=1}^{L}\left(e^{t^{\frac{1}{3}} x_{i}} \vee e^{n t^{\frac{1}{3}} x_{i}}\right)=C_{1}^{L} \prod_{i=1}^{L}\left(e^{t^{\frac{1}{3}} x_{i}} \vee e^{n t^{\frac{1}{3}} x_{i}}\right)\left(\sum_{\vec{m} \in \mathfrak{M}(L, n)}\left(\begin{array}{c}
n \\
\vec{m}
\end{array}\right)\right)
$$

It suffices to show that there exists a constant $C_{2}$ such that for all $L \in \mathbb{Z}_{\geq 1}$

$$
\sum_{\vec{m} \in \mathfrak{M}(L, n)}\left(\begin{array}{c}
n \\
\vec{m}
\end{array}\right) \leq C_{2}^{L} .
$$

Once this shown, by (4.5) and triangle inequality, we see that $\left|\partial_{s}^{n}\left(\prod_{i=1}^{L} \phi_{s, T}\left(x_{i}\right)\right)\right|$ is upper bounded by $\left(C_{1} C_{2}\right)^{L} \prod_{i=1}^{L}\left(e^{t^{\frac{1}{3}} x_{i}} \vee e^{n t^{\frac{1}{3}} x_{i}}\right)$. Applying Proposition 4.1 yields

$$
\left|\int_{\mathbb{R}^{L}} \operatorname{Pf}\left[K\left(x_{i}, x_{j}\right)\right]_{i, j=1}^{L} \partial_{s}^{n}\left(\prod_{i=1}^{L} \phi_{s, t}\left(x_{i}\right)\right) d x\right| \leq(2 L)^{\frac{L}{2}} C^{L}\left(\int_{\mathbb{R}} F_{\frac{1}{3}, 2}(x)\left(e^{t^{\frac{1}{3}} x} \vee e^{n t^{\frac{1}{3}} x}\right) d x\right)^{L} .
$$

Since $t$ is fixed, the integrand on the right hand side above decays super-exponentially at $+\infty$ and exponentially at $-\infty$, hence is integrable. The value of the integration is a constant that only depends on $n, t$. This completes our proof of the lemma.

It remains to prove (4.6). Let $\# A$ be the number of elements in $A$. Note that

$$
\# \mathfrak{M}(L, n)=\#\left\{\vec{m}=\left(m_{1}, \ldots, m_{L}\right) \in \mathbb{Z}_{\geq 0}^{L}, \sum_{i=1}^{L} m_{i}=n\right\} \leq L \# \mathfrak{M}(L, n-1) .
$$


Iterating this inequality yields $\# \mathfrak{M}(L, n) \leq L^{n}$. In addition, for each $\vec{m} \in \mathfrak{M}(L, n),\left(\begin{array}{c}n \\ \vec{m}\end{array}\right)$ is upper bounded by $n$ !. We can find a large constant $C_{2}=C_{2}(n)$ such that for all $L \geq 1$,

$$
\sum_{\vec{m} \in \mathfrak{M}(L, n)}\left(\begin{array}{c}
n \\
\vec{m}
\end{array}\right) \leq n ! \# \mathfrak{M}(L, n) \leq n ! L^{n} \leq C_{2}^{L} .
$$

The next two propositions validate that we can interchange the order of derivative, summation and integral.

Proposition 4.5. For every fixed $n, L \in \mathbb{Z}_{\geq 1}, s \in[0,1]$ and $t>0$, we have

$$
\partial_{s}^{n} \int_{\mathbb{R}^{L}} \operatorname{Pf}\left[K\left(x_{i}, x_{j}\right)\right]_{i, j=1}^{L} \prod_{i=1}^{L} \phi_{s, t}\left(x_{i}\right) d x_{i}=\int_{\mathbb{R}^{L}} \operatorname{Pf}\left[K\left(x_{i}, x_{j}\right)\right]_{i, j=1}^{L} \partial_{s}^{n}\left(\prod_{i=1}^{L} \phi_{s, t}\left(x_{i}\right)\right) d x_{1} \ldots d x_{L}
$$

Proof. The proof follows from the dominated convergence theorem. It suffices to show that we can find an integrable function $G\left(x_{1}, \ldots, x_{L}\right)$ such that for all $s \in[0,1]$,

$$
\left|\operatorname{Pf}\left[K\left(x_{i}, x_{j}\right)\right]_{i, j=1}^{L} \partial_{s}^{n}\left(\prod_{i=1}^{L} \phi_{s, t}\left(x_{i}\right)\right)\right| \leq G\left(x_{1}, \ldots, x_{L}\right)
$$

By the argument in the proof of Lemma 4.4, we can take

$$
G\left(x_{1}, \ldots, x_{L}\right)=(2 L)^{\frac{L}{2}} C^{L} \prod_{i=1}^{L} F_{\frac{1}{3}, 2}\left(x_{i}\right)\left(e^{t^{\frac{1}{3}} x_{i}} \vee e^{n t^{\frac{1}{3}} x_{i}}\right)
$$

where $C$ is the constant in Lemma 4.4. Since $G$ is integrable and satisfy (4.8), this completes our proof.

Proposition 4.6. For fixed $n \in \mathbb{Z}_{\geq 0}, t>0$ and $s \in[0,1]$ we have the following interchange of differentiation and summation holds

$$
\partial_{s}^{n}\left(\sum_{L=1}^{\infty} \frac{1}{L !} \int_{\mathbb{R}^{L}} \operatorname{Pf}\left[K\left(x_{i}, x_{j}\right)\right]_{i, j=1}^{L} \prod_{i=1}^{L} \phi_{s, t}\left(x_{i}\right) d x_{i}\right)=\sum_{L=1}^{\infty} \partial_{s}^{n}\left(\int_{\mathbb{R}^{L}} \operatorname{Pf}\left[K\left(x_{i}, x_{j}\right)\right]_{i, j=1}^{L} \prod_{i=1}^{L} \phi_{s, t}\left(x_{i}\right) d x_{i}\right) .
$$

Proof. A sufficient condition for the interchange of the order of derivative and infinite summation is that (see [DT19, Proposition 4.2]),

(i) $\sum_{L=1}^{\infty} \frac{1}{L !} \int_{\mathbb{R}^{L}} \operatorname{Pf}\left[K\left(x_{i}, x_{j}\right)\right]_{i, j=1}^{L} \prod_{i=1}^{L} \phi_{s, t}\left(x_{i}\right) d x_{i}$ converges pointwisely for $s \in[0,1]$.

(ii) For all $n \in \mathbb{Z}_{\geq 1}, \sum_{L=1}^{\infty} \frac{1}{L !} \partial_{s}^{n}\left(\int_{\mathbb{R}^{L}} \operatorname{Pf}\left[K\left(x_{i}, x_{j}\right)\right]_{i, j=1}^{L} \prod_{i=1}^{L} \phi_{s, t}\left(x_{i}\right) d x_{i}\right)$ converges uniformly for $s \in[0,1]$.

Applying Proposition 4.5, for (ii), we can place $\partial_{s}^{n}$ inside the integral. Both (i) and (ii) then follow from Lemma 4.4 and the convergence of $\sum_{L=1}^{\infty} \frac{C^{L}(2 L)^{\frac{L}{2}}}{L !}$.

Proof of Lemma 2.7. It is enough to show (2.9). Combining Proposition 4.5 and 4.6, we know that

$$
\begin{aligned}
& \int_{0}^{1} s^{-\alpha} \partial_{s}^{n}\left(\sum_{L=1}^{\infty} \int_{\mathbb{R}^{L}} \operatorname{Pf}\left[K\left(x_{i}, x_{j}\right)\right]_{i, j=1}^{L} \prod_{i=1}^{L} \phi_{s, t}\left(x_{i}\right) d x_{i}\right) d s \\
& =\int_{0}^{1} s^{-\alpha}\left(\sum_{L=1}^{\infty} \int_{\mathbb{R}^{L}} \operatorname{Pf}\left[K\left(x_{i}, x_{j}\right)\right]_{i, j=1}^{L} \partial_{s}^{n}\left(\prod_{i=1}^{L} \phi_{s, t}\left(x_{i}\right)\right) d x_{1} \ldots d x_{L}\right) d s
\end{aligned}
$$

Thus, what remains to prove is that

$$
\begin{aligned}
& \int_{0}^{1} s^{-\alpha}\left(\sum_{L=1}^{\infty} \int_{\mathbb{R}^{L}} \operatorname{Pf}\left[K\left(x_{i}, x_{j}\right)\right]_{i, j=1}^{L} \partial_{s}^{n}\left(\prod_{i=1}^{L} \phi_{s, t}\left(x_{i}\right)\right) d x_{1} \ldots d x_{L}\right) d s \\
& =\sum_{L=1}^{\infty} \int_{0}^{1} s^{-\alpha}\left(\int_{\mathbb{R}^{L}} \operatorname{Pf}\left[K\left(x_{i}, x_{j}\right)\right]_{i, j=1}^{L} \partial_{s}^{n}\left(\prod_{i=1}^{L} \phi_{s, t}\left(x_{i}\right)\right) d x_{1} \ldots d x_{L}\right) d s
\end{aligned}
$$

This is justified via the dominated convergence theorem, using Lemma 4.4 and the convergence of $\sum_{L=1}^{\infty} \frac{C^{L}(2 L)^{\frac{L}{2}}}{L !}$. 


\section{Proof of Proposition 2.9}

In this section, we prove Proposition 2.9. The main inputs will be Proposition 4.1 and 5.2.

Define $V_{n}(x)=n x-\frac{1}{3} x^{\frac{3}{2}}$ and $U_{n}(x)=n x-\frac{2}{3} x^{3 / 2}$. By straightforward calculus, $V_{n}\left(\sigma \wedge 4 n^{2}\right)\left(\right.$ resp. $\left.U_{n}\left(\sigma \wedge n^{2}\right)\right)$ is the maximum of $V_{n}$ (resp. $U_{n}$ ) over $x \in[0, \sigma]$. Recall from the beginning of Section 4 that $F_{\alpha, \beta}(x)=$ $e^{-\alpha x^{\frac{3}{2}}} \mathbf{1}_{\{x \geq 0\}}+(1-x)^{\beta} \mathbf{1}_{\{x<0\}}$. We start with the following lemma.

Lemma 5.1. Fix $n \in \mathbb{Z}_{\geq 1}$ and $t_{0}>0$, there exists $C=C\left(n, t_{0}\right)$ such that for all $\sigma \geq 0$ and $t>t_{0}$,

(i) $\int_{-\infty}^{\sigma} \exp (\operatorname{tn} x) F_{\frac{1}{3}, 2}\left(t^{\frac{2}{3}} x\right) d x \leq C t^{-\frac{1}{2}} \exp \left(t V_{n}\left(\sigma \wedge 4 n^{2}\right)\right)$

(ii) $\int_{-\infty}^{\sigma} \exp (t n x) F_{\frac{2}{3}, 2}\left(t^{\frac{2}{3}} x\right) \leq C t^{-\frac{1}{2}} \exp \left(t U_{n}\left(\sigma \wedge n^{2}\right)\right)$

Proof. Let us first demonstrate (i). Decompose

$$
\int_{-\infty}^{\sigma} \exp (\operatorname{tn} x) F_{\frac{1}{3}, 2}\left(t^{\frac{2}{3}} x\right) d x=\int_{-\infty}^{0} \exp (\operatorname{tn} x) F_{\frac{1}{3}, 2}\left(t^{\frac{2}{3}} x\right) d x+\int_{0}^{\sigma} \exp (\operatorname{tn} x) F_{\frac{1}{3}, 2}\left(t^{\frac{2}{3}} x\right) d x
$$

Since $F_{\alpha, \beta}(x)$ equals $(1-x)^{\beta}$ when $x$ is negative, we can rewrite the first term on the right hand side in the above display into $\int_{-\infty}^{0} \exp (\operatorname{tn} x)\left(1-t^{\frac{2}{3}} x\right)^{2} d x$. We have

$$
\int_{-\infty}^{0} e^{t n x}\left(1-t^{\frac{2}{3}} x\right)^{2} d x=t^{-1} \int_{0}^{\infty} e^{-n x}\left(1+t^{-\frac{1}{3}} x\right)^{2} d x \leq t^{-1} \int_{0}^{\infty} e^{-n x}\left(1+t_{0}^{-1 / 3} x\right)^{2} d x \leq C t^{-1},
$$

where the first equality is due to a change of variable $x \rightarrow-t^{-1} x$, the second equality follows our condition $t \geq t_{0}$ and the third is due to $\int_{0}^{\infty} e^{-n x}\left(1+t_{0}^{-\frac{1}{3} x}\right)^{2} d x$ is finite constant only depending on $n, t_{0}$. We have shown that the first term on the right hand side of (5.1) is upper bounded by $C t^{-1}$ for some $C=C\left(n, t_{0}\right)$. Since $V_{n}\left(\sigma \wedge 4 n^{2}\right)$ is non-negative, which implies that $t^{-1} \leq C t^{-\frac{1}{2}} \exp \left(t V_{n}\left(\sigma \wedge 4 n^{2}\right)\right)$ for $t \geq t_{0}$. To prove (i), it suffices to prove the second term on the right hand side of (5.1) is also upper bounded by $C t^{-\frac{1}{2}} \exp \left(t V_{n}\left(\sigma \wedge 4 n^{2}\right)\right)$. Note that when $x \geq 0, F_{\frac{1}{3}, 2}\left(t^{\frac{2}{3}} x\right)=\exp \left(-\frac{1}{3} t x^{\frac{3}{2}}\right)$ which yields $\int_{0}^{\sigma} \exp (t n x) F_{\frac{1}{3}, 2}\left(t^{\frac{2}{3}} x\right) d x=\int_{0}^{\sigma} \exp \left(t V_{n}(x)\right) d x$. Thus we only need to show that there exists a constant $C=C\left(n, t_{0}\right)$ such that for $t>t_{0}$

$$
\int_{0}^{\sigma} e^{t V_{n}(x)} d x \leq C t^{-\frac{1}{2}} e^{t V_{n}\left(\sigma \wedge 4 n^{2}\right)}
$$

To this aim, we split our discussion into the following three cases.

Case 1. $\sigma \in\left[0, n^{2}\right]$. Since $V_{n}(x)$ is concave on $\sigma \in\left[0, n^{2}\right]$. Hence, for all $x \in[0, \sigma]$,

$$
V_{n}(x) \leq V_{n}(\sigma)+V_{n}^{\prime}(\sigma)(x-\sigma)=V_{n}(\sigma)+\left(n-\frac{1}{2} \sqrt{\sigma}\right)(x-\sigma) \leq V_{n}(\sigma)+\frac{n}{2}(x-\sigma) .
$$

The last inequality above follows since $x \leq \sigma$ and $n-\frac{1}{2} \sqrt{\sigma} \geq n / 2$. Using the displayed inequality above,

$$
\int_{0}^{\sigma} e^{t V_{n}(x)} d x \leq \int_{0}^{\sigma} e^{t\left(V_{n}(\sigma)+\frac{n}{2}(x-\sigma)\right)} d x=e^{t V_{n}(\sigma)} \int_{0}^{\sigma} e^{\frac{n t}{2}(x-\sigma)} d x \leq \frac{2}{n t} e^{t V_{n}(\sigma)} .
$$

which implies (5.2).

Case 2. $\sigma \in\left[n^{2}, 4 n^{2}\right]$. First via a change of variable $x=r^{2}, \int_{0}^{\sigma} e^{t V_{n}(x)} d x=\int_{0}^{\sqrt{\sigma}} 2 r e^{t V_{n}\left(r^{2}\right)} d r$. Therefore, we only need to prove (5.2) with $\int_{0}^{\sqrt{\sigma}} 2 r e^{t V_{n}\left(r^{2}\right)} d r$ in place of $\int_{0}^{\sigma} e^{t V_{n}(x)} d x$. Since $r \leq \sqrt{\sigma} \leq 2 n$,

$$
V_{n}\left(r^{2}\right)-V_{n}(\sigma)=n\left(r^{2}-\sigma\right)-\frac{1}{3}\left(r^{3}-\sigma^{\frac{3}{2}}\right) \leq \frac{\sqrt{\sigma}}{2}\left(r^{2}-\sigma\right)-\frac{1}{3}\left(r^{3}-\sigma^{\frac{3}{2}}\right)=-\frac{1}{3}(r-\sqrt{\sigma})^{2}\left(r+\frac{1}{2} \sqrt{\sigma}\right) .
$$

Since $\sigma \geq n^{2}$ and $r \geq 0$, from the above displayed inequality, $V_{n}\left(r^{2}\right) \leq V_{n}(\sigma)-\frac{n}{6}(r-\sqrt{\sigma})^{2}$. Consequently,

$$
\int_{0}^{\sqrt{\sigma}} 2 r e^{t V_{n}\left(r^{2}\right)} d r \leq \int_{0}^{\sqrt{\sigma}} 2 r e^{t V_{n}(\sigma)} e^{-\frac{t n}{6}(r-\sqrt{\sigma})^{2}} d r=e^{t V_{n}(\sigma)} \int_{0}^{\sqrt{\sigma}} 2 r e^{-\frac{t}{6}(r-\sqrt{\sigma})^{2}} d r \leq \frac{C}{\sqrt{t}} e^{t V_{n}(\sigma)} .
$$

This completes the proof of (5.2). In the last inequality above, we used $\sqrt{\sigma} \leq 2 n$ and $t \geq t_{0}$, which implies that

$$
\int_{0}^{\sqrt{\sigma}} 2 r e^{-\frac{t}{6}(r-\sqrt{\sigma})^{2}} d r \leq \int_{-\infty}^{\infty} 2(r+\sqrt{\sigma}) e^{-\frac{t}{6} r^{2}} d r \leq \int_{-\infty}^{\infty} 2(r+2 n) e^{-\frac{t}{6} r^{2}} d r=C_{1} t^{-1}+C_{2} t^{-1 / 2} \leq C t^{-1 / 2} .
$$


Case 3. $\sigma>4 n^{2}$. As illustrated in Case 2, we only need to show that for $t>t_{0}$,

$$
\int_{0}^{\sqrt{\sigma}} 2 r e^{t V_{n}\left(r^{2}\right)} d r \leq C t^{-\frac{1}{2}} e^{t V_{n}\left(\sigma \wedge 4 n^{2}\right)}
$$

Note that $V_{n}\left(r^{2}\right)-\frac{4}{3} n^{3}=n r^{2}-\frac{1}{3} r^{3}-\frac{4}{3} n^{3}=-\frac{1}{3}(r-2 n)^{2}(n+r) \leq-\frac{n}{3}(r-2 n)^{2}$. This implies

$$
\int_{0}^{\sqrt{\sigma}} 2 r e^{t V_{n}\left(r^{2}\right)} d r \leq \int_{0}^{\sqrt{\sigma}} 2 r e^{\frac{4}{3} t n^{3}} e^{-\frac{n}{3} t(r-2 n)^{2}} d r \leq e^{\frac{4}{3} n^{3} t} \int_{0}^{\sqrt{\sigma}} 2 r e^{-\frac{n}{3} t(r-2 n)^{2}} d r \leq \frac{C}{\sqrt{t}} e^{\frac{4}{3} n^{3} t} .
$$

The last inequality is due to a similar argument as in Case $\mathbf{2}$ above. Since $\sigma>4 n^{2}$, we have $V_{n}\left(\sigma \wedge 4 n^{2}\right)=$ $V_{n}\left(4 n^{2}\right)=\frac{4}{3} n^{3}$, thus we showed (5.2). So far, we complete the proof of (i).

The proof of (ii) will be rather similar, instead of (5.2), the key step is to show that there exists $C=C\left(n, t_{0}\right)$ such that for $t \geq t_{0}, \int_{0}^{\sigma} \exp \left(t U_{n}(x)\right) d x \leq C t^{-\frac{1}{2}} \exp \left(t U_{n}\left(\sigma \wedge n^{2}\right)\right)$. We skip the details.

Proposition 5.2. For fixed $n \in \mathbb{Z}_{\geq 1}$ and $t_{0}>0$, there exists constant $C=C\left(n, t_{0}\right)$ such that for every $\sigma \geq 0$ and $t>t_{0}$,

(a) $\int_{-\infty}^{\infty}\left|\phi_{e^{-t \sigma, t}}(x)\right| F_{\frac{1}{3}, 2}(x) d x \leq C t^{\frac{1}{6}} \exp \left(t V_{1}(\sigma \wedge 4)-t \sigma\right)$

(b) $\int_{-\infty}^{\infty}\left|\phi_{e^{-t \sigma, t}}^{(n)}(x)\right| F_{\frac{1}{3}, 2}(x) d x \leq C t^{\frac{1}{6}} \exp \left(t V_{n}\left(\sigma \wedge 4 n^{2}\right)\right)$

(c) $\int_{-\infty}^{\infty}\left|\phi_{e^{-t \sigma, t}}(x)\right| F_{\frac{2}{3}, 2}(x) d x \leq C t^{\frac{1}{6}} \exp \left(t U_{1}(\sigma \wedge 1)-t \sigma\right)$

(d) $\int_{-\infty}^{\infty}\left|\phi_{e^{-t \sigma, t}}^{(n)}(x)\right| F_{\frac{2}{3}, 2}(x) d x \leq C t^{\frac{1}{6}} \exp \left(t U_{n}\left(\sigma \wedge n^{2}\right)\right)$.

Proof. We first prove (a). Via a change of variable $x \rightarrow t^{\frac{2}{3}} x$,

$$
\int_{-\infty}^{\infty}\left|\phi_{e^{-t \sigma}, t}(x)\right| F_{\frac{1}{3}, 2}(x) d x=t^{\frac{2}{3}} \int_{-\infty}^{\infty}\left|\phi_{e^{-t \sigma}, t}\left(t^{\frac{2}{3}} x\right)\right| F_{\frac{1}{3}, 2}\left(t^{\frac{2}{3}} x\right) d x .
$$

We decompose the integral region on the right hand side above into $(-\infty, \sigma) \cup(\sigma, \infty)$ and write

$$
\int_{-\infty}^{\infty}\left|\phi_{e^{-t \sigma}, t}\left(t^{\frac{2}{3}} x\right)\right| F_{\frac{1}{3}, 2}\left(t^{\frac{2}{3}} x\right) d x=\left(\int_{-\infty}^{\sigma}+\int_{\sigma}^{\infty}\right)\left|\phi_{e^{-t \sigma}, t}\left(t^{\frac{2}{3}} x\right)\right| F_{\frac{1}{3}, 2}\left(t^{\frac{2}{3}} x\right) d x=\mathrm{E}_{1}+\mathrm{E}_{2}
$$

We provide upper bounds for $\mathrm{E}_{1}$ and $\mathrm{E}_{2}$ respectively. By Lemma $4.3,\left|\phi_{e^{-t \sigma}, t}\left(t^{\frac{2}{3}} x\right)\right| \leq 2 \exp (t(x-\sigma))$, so

$$
\mathrm{E}_{1} \leq 2 e^{-t \sigma} \int_{-\infty}^{\sigma} e^{t x} F_{\frac{1}{3}, 2}(x) d x \leq C t^{-\frac{1}{2}} e^{t V_{1}(\sigma \wedge 4)-t \sigma}
$$

where the last inequality above is due to Lemma 5.1 (i) (setting $n=1$ therein). On the other hand, since $F_{\frac{1}{3}, 2}\left(t^{\frac{2}{3}} x\right)=\exp \left(-\frac{1}{3} t x^{\frac{3}{2}}\right)$ when $x \geq 0$,

$$
\mathrm{E}_{2}=\int_{\sigma}^{\infty}\left|\phi_{e^{-t \sigma}, t}\left(t^{\frac{2}{3}} x\right)\right| e^{-\frac{1}{3} t x^{3 / 2}} d x \leq \int_{\sigma}^{\infty} e^{-\frac{1}{3} t x^{3 / 2}} d x=t^{-\frac{2}{3}} \int_{t^{\frac{2}{3}} \sigma}^{\infty} e^{-\frac{1}{3} x^{3 / 2}} d x \leq C t^{-\frac{2}{3}} e^{-\frac{1}{3} t \sigma^{\frac{3}{2}}}
$$

The first inequality above is due to Lemma 4.3, which yields $\left|\phi_{e^{-t \sigma}, t}\left(t^{\frac{2}{3}} x\right)\right| \leq 1$. The second inequality follows from a change of variable $x \rightarrow t^{-\frac{2}{3}} \sigma$, and the last inequality is due to the fact $\int_{y}^{\infty} \exp \left(-\frac{1}{3} x^{\frac{3}{2}}\right) d x \leq C \exp \left(-\frac{1}{3} y^{\frac{3}{2}}\right)$, which holds for all $y \geq 0$. Combining (5.3) and (5.4) and recall that $\int_{-\infty}^{\infty} \phi_{e^{-t \sigma, t},}(x) F_{\frac{1}{3}, 2}(x) d x=t^{\frac{2}{3}}\left(\mathrm{E}_{1}+\mathrm{E}_{2}\right)$, we obtain

$$
\int_{-\infty}^{\infty}\left|\phi_{e^{-t \sigma}, t}(x)\right| F_{\frac{1}{3}, 2}(x) d x \leq C t^{\frac{2}{3}}\left(t^{-\frac{1}{2}} \exp \left(t V_{1}(\sigma \wedge 4)-t \sigma\right)+t^{-\frac{2}{3}} e^{-\frac{1}{3} t \sigma^{\frac{3}{2}}}\right) .
$$

Since $V_{1}(\sigma \wedge 4)$ is the maximum of $V_{1}(x)$ for $x \in[0, \sigma], V_{1}(\sigma \wedge 4)-\sigma \geq V_{1}(\sigma)-\sigma=-\frac{1}{3} \sigma^{\frac{3}{2}}$, so the first term on the right hand side above dominates, this completes the proof of (a).

For the proof of (b), via a change of variable $x \rightarrow t^{\frac{2}{3}} x$,

$$
\int_{-\infty}^{\infty}\left|\phi_{e^{-t \sigma, t}}^{(n)}(x)\right| F_{\frac{1}{3}, 2}(x) d x=t^{\frac{2}{3}} \int_{-\infty}^{\infty}\left|\phi_{e^{-t \sigma, t}}^{(n)}\left(t^{\frac{2}{3}} x\right)\right| F_{\frac{1}{3}, 2}\left(t^{\frac{2}{3}} x\right) d x
$$

Decompose the integral on the right hand side of the above display as

$$
\int_{-\infty}^{\infty}\left|\phi_{e^{-t \sigma}, t}^{(n)}\left(t^{\frac{2}{3}} x\right)\right| F_{\frac{1}{3}, 2}\left(t^{\frac{2}{3}} x\right) d x=\left(\int_{-\infty}^{\sigma}+\int_{\sigma}^{\infty}\right)\left|\phi_{e^{-t \sigma}, t}^{(n)}\left(t^{\frac{2}{3}} x\right)\right| F_{\frac{1}{3}, 2}\left(t^{\frac{2}{3}} x\right) d x=\mathrm{E}_{1}^{\prime}+\mathrm{E}_{2}^{\prime} .
$$


Let us upper bound $\mathrm{E}_{1}^{\prime}$ and $\mathrm{E}_{2}^{\prime}$ respectively. By Lemma 4.3, we know that $\left|\phi_{e^{-t \sigma}, t}^{(n)}\left(t^{\frac{2}{3}} x\right)\right| \leq C \exp (n t x)$. Using this together with Lemma 5.1 (i), we get

$$
\mathrm{E}_{1}^{\prime} \leq C \int_{-\infty}^{\sigma} e^{n t x} F_{\frac{1}{3}, 2}\left(t^{\frac{2}{3}} x\right) d x \leq C t^{-\frac{1}{2}} \exp \left(t V_{n}\left(\sigma \wedge 4 n^{2}\right)\right)
$$

For $\mathrm{E}_{2}^{\prime}$, note that $F_{\frac{1}{3}, 2}\left(t^{\frac{2}{3}} x\right)$ simplifies to $\exp \left(-\frac{1}{3} t x^{\frac{3}{2}}\right)$ for $x \geq 0$. By Lemma $4.3,\left|\phi_{e^{-t \sigma}, t}^{(n)}\left(t^{\frac{2}{3}} x\right)\right| \leq C \exp (n t \sigma)$. Using this inequality implies

$$
\mathrm{E}_{2}^{\prime}=\int_{\sigma}^{\infty} \phi_{e^{-t \sigma}, t}^{(n)}\left(t^{\frac{2}{3}} x\right) e^{-\frac{1}{3} t x^{\frac{3}{2}}} d x \leq C e^{t n \sigma} \int_{\sigma}^{\infty} e^{-\frac{1}{3} t x^{\frac{3}{2}}} d x \leq C t^{-\frac{2}{3}} e^{t n \sigma-\frac{1}{3} t \sigma^{\frac{3}{2}}} .
$$

Recall that $\int_{-\infty}^{\infty}\left|\phi_{e^{-t \sigma}, t}^{(n)}(x)\right| F_{\frac{1}{3}, 2}(x) d x=t^{\frac{2}{3}}\left(\mathrm{E}_{1}^{\prime}+\mathrm{E}_{2}^{\prime}\right)$, combining (5.5) and (5.6) yields

$$
\int_{-\infty}^{\infty}\left|\phi_{e^{-t \sigma}, t}^{(n)}(x)\right| F_{\frac{1}{3}, 2}(x) d x \leq C t^{\frac{2}{3}}\left(t^{-\frac{1}{2}} e^{t V_{n}\left(\sigma \wedge 4 n^{2}\right)}+t^{-\frac{2}{3}} e^{t\left(n \sigma-\frac{1}{3} \sigma^{\frac{3}{2}}\right)}\right) \leq C t^{\frac{1}{6}} \exp \left(t V_{n}\left(\sigma \wedge 4 n^{2}\right)\right) .
$$

The last inequality above is due to $V_{n}\left(\sigma \wedge 4 n^{2}\right) \geq V_{n}(\sigma)=n \sigma-\frac{1}{3} \sigma^{\frac{3}{2}}$. This completes the proof of (b).

The proof for (c), (d) follows a rather similar argument as for (a), (b). Instead of using Lemma 5.1 (i), one needs tp Lemma 5.1 (ii). We omit the details here.

For any $L \geq 2$ and $\vec{m}=\left(m_{1}, \ldots, m_{L}\right) \in \mathfrak{M}(L, n)$, denote by

$$
\mathcal{I}_{\vec{m}}=\int_{0}^{1} s^{-\alpha} d s \int_{\mathbb{R}^{L}} \operatorname{Pf}\left[K\left(x_{i}, x_{j}\right)\right]_{i, j=1}^{L} \prod_{i=1}^{L} \phi_{s, t}^{\left(m_{i}\right)}\left(x_{i}\right) d x_{i} .
$$

Proposition 5.3. Fix $p \in \mathbb{Z}_{\geq 0}$ and $t_{0}>0$. Recall that $n=\lfloor p\rfloor+1$ and $\alpha=p+1-n$. Define $\delta_{p}=\min \left(\frac{2}{3}, \frac{p^{3}}{4}\right)$. There exists $C=C\left(n, t_{0}\right)$ such that for all $L \geq 2, t \geq t_{0}$ and $\vec{m} \in \mathfrak{M}(L, n)$, we have $\left|\mathcal{I}_{\vec{m}}\right| \leq C^{L}(2 L)^{\frac{L}{2}} t^{\frac{L}{6}} e^{\frac{p^{3}}{3} t-\delta_{p} t}$.

Proof. Without loss of generality, we assume that $m_{1}, \ldots, m_{r}>0$ and $m_{r+1}=\cdots=m_{L}=0$, note that $1 \leq r \leq n \wedge L$. Referring to (5.7), by a change of variable $s=e^{-t \sigma}$, we obtain

$$
\mathcal{I}_{\vec{m}}=\int_{0}^{\infty} e^{t(\alpha-1) \sigma} d \sigma \int_{\mathbb{R}^{L}} \operatorname{Pf}\left[K\left(x_{i}, x_{j}\right)\right]_{i, j=1}^{L} \prod_{i=1}^{L} \phi_{e^{-t \sigma}, t}^{\left(m_{i}\right)}\left(x_{i}\right) d x_{i} .
$$

It suffice to show that the right hand side of the above display is upper bounded by $C^{L}(2 L)^{\frac{L}{2}} t^{\frac{L}{6}} e^{\frac{p^{3} t}{3}-\delta_{p} t}$. We divide our argument into two stages. We prove the inequality for $L \geq 2 n^{3}$ in Stage 1 and Stage 2 will cover the case $2 \leq L<2 n^{3}$.

Stage 1. $L \geq 2 n^{3}$. Via Proposition 4.1 (i), $\left|\operatorname{Pf}\left[K\left(x_{i}, x_{j}\right)\right]_{i, j=1}^{L}\right|$ is upper bounded by $C^{L}(2 L)^{\frac{L}{2}} \prod_{i=1}^{L} F_{\frac{1}{3}, 2}\left(x_{i}\right)$, thus

$$
\left|\mathcal{I}_{\vec{m}}\right| \leq C^{L}(2 L)^{\frac{L}{2}} \int_{0}^{\infty} e^{t(\alpha-1) \sigma} \prod_{i=1}^{L}\left(\int_{\mathbb{R}} F_{\frac{1}{3}, 2}(x)\left|\phi_{e^{-t \sigma}, t}^{\left(m_{i}\right)}(x)\right| d x\right) d \sigma .
$$

Applying Proposition 5.2 (a) and (b), there exists a constant $C=C\left(n, t_{0}\right)$ such that for $t>t_{0}$,

$$
\int_{\mathbb{R}} F_{\frac{1}{3}, 2}(x)\left|\phi_{e^{-t \sigma}, t}^{\left(m_{i}\right)}(x)\right| d x \leq \begin{cases}C t^{\frac{1}{6}} \exp \left(t V_{m_{i}}\left(\sigma \wedge 4 m_{i}^{2}\right)-t \sigma\right) & i \leq r \\ C t^{\frac{1}{6}} \exp \left(t V_{1}(\sigma \wedge 4)-t \sigma\right) & i>r .\end{cases}
$$

Applying this inequality to the right hand side of (5.8), we find that $\left|\mathcal{I}_{\vec{m}}\right| \leq C^{L}(2 L)^{\frac{L}{2}} t^{\frac{L}{6}} \int_{0}^{\infty} e^{t \mathrm{M}_{1}(\sigma)} d \sigma$, where

$$
\mathrm{M}_{1}(\sigma)=(\alpha-1) \sigma+\sum_{i=1}^{r} V_{m_{i}}\left(\sigma \wedge 4 m_{i}^{2}\right)+(L-r)\left(V_{1}(\sigma \wedge 4)-\sigma\right) .
$$

To prove Proposition 5.3, it suffices to show that for there exists $C=C\left(n, t_{0}\right)$, such that for all $t \geq t_{0}$ and $\vec{m} \in \mathfrak{M}(L, n)$,

$$
\int_{0}^{\infty} e^{t \mathrm{M}_{1}(\sigma)} d \sigma \leq C e^{\frac{p^{3}}{3} t-\delta_{p} t}
$$


where $\delta_{p}=\min \left(\frac{2}{3}, \frac{p^{3}}{4}\right)$. To this aim, we decompose

$$
\int_{0}^{\infty} e^{t \mathrm{M}_{1}(\sigma)} d \sigma=\int_{0}^{4} e^{t \mathrm{M}_{1}(\sigma)} d \sigma+\int_{4}^{\infty} e^{t \mathrm{M}_{1}(\sigma)} d \sigma=\mathcal{J}_{1}+\mathcal{J}_{2}
$$

For $\mathcal{J}_{1}$, since $\sigma \leq 4, \mathrm{M}_{1}$ simplifies to

$$
\begin{aligned}
\mathrm{M}_{1}(\sigma) & =(\alpha-1) \sigma+\sum_{i=1}^{r} V_{m_{i}}(\sigma)+(L-r)\left(V_{1}(\sigma)-\sigma\right) \\
& =(\alpha-1)+\sum_{i=1}^{r}\left(m_{i} \sigma-\frac{1}{3} \sigma^{\frac{3}{2}}\right)-\frac{1}{3}(L-r) \sigma^{\frac{3}{2}}=p \sigma-\frac{L}{3} \sigma^{\frac{3}{2}} .
\end{aligned}
$$

The last equality is due to $\sum_{i=1}^{r} m_{i}+\alpha-1=n+\alpha-1=p$. Since $L \geq 2 n^{3} \geq 2, \mathrm{M}_{1}(\sigma)=p \sigma-\frac{2 L}{3} \sigma^{\frac{3}{2}} \leq$ $p \sigma-\frac{4}{3} \sigma^{\frac{3}{2}} \leq \frac{p^{3}}{12}$. We find that

$$
\mathcal{J}_{1}=\int_{0}^{4} e^{t \mathrm{M}_{1}(\sigma)} d \sigma \leq \int_{0}^{4} e^{\frac{p^{3} t}{12}} d \sigma \leq 4 e^{\frac{p^{3} t}{12}}
$$

For $\mathcal{J}_{2}$, since $\sigma \geq 4, V_{1}(\sigma \wedge 4)=V_{1}(4)=\frac{4}{3}$. Moreover, the maximum of $V_{m_{i}}(\sigma)=m_{i} \sigma-\frac{1}{3} \sigma^{\frac{3}{2}}$ equals $\frac{4}{3} m_{i}^{3}$, hence $V_{m_{i}}\left(\sigma \wedge 4 m_{i}^{2}\right) \leq \frac{4}{3} m_{i}^{3}$. As a result,

$$
\mathrm{M}_{1}(\sigma) \leq(\alpha-1) \sigma+\frac{4}{3} \sum_{i=1}^{r} m_{i}^{3}+(L-r)\left(\frac{4}{3}-\sigma\right) \leq(\alpha-1) \sigma+\frac{4}{3} n^{3}-(L-r) \frac{8}{3} .
$$

The last inequality follows from the fact that $\sum_{i=1}^{r} m_{i}^{3} \leq\left(\sum_{i=1}^{r} m_{i}\right)^{3}=n^{3}$ and $\frac{4}{3}-\sigma \leq-\frac{8}{3}$. Note that $r$ is the number of $m_{i}$ which is non-zero, so $r \leq n$. Moreover, since $L \geq 2 n^{3}$,

$$
\mathrm{M}_{1}(\sigma) \leq \frac{4}{3} n^{3}-\left(2 n^{3}-n\right) \frac{8}{3}+(\alpha-1) \sigma \leq(\alpha-1) \sigma
$$

Consequently, we have $\mathcal{J}_{2}=\int_{4}^{\infty} e^{t \mathrm{M}_{1}(\sigma)} d \sigma \leq \int_{0}^{\infty} e^{(\alpha-1) \sigma t} d \sigma=\frac{t^{-1}}{1-\alpha}$. Combining this with (5.10) yields that for all $t>t_{0}$, (note that $\frac{p^{3}}{12} \leq \frac{p^{3}}{3}-\delta_{p}$ )

$$
\int_{0}^{\infty} e^{t \mathrm{M}_{1}(\sigma)} d \sigma=\mathcal{J}_{1}+\mathcal{J}_{2} \leq 4 e^{\frac{p^{3} t}{12}}+\frac{t^{-1}}{1-\alpha} \leq C e^{\frac{p^{3}}{3} t-\delta_{p} t}
$$

We prove the desired (5.9) and conclude our proof for Stage 1.

Stage 2. $2 \leq L \leq 2 n^{3}$. Via Proposition 4.1 (ii), $\left|\operatorname{Pf}\left[K\left(x_{i}, x_{j}\right)\right]_{i, j=1}^{L}\right|$ is bounded by $C^{L} \prod_{i=1}^{L} F_{\frac{2}{3}, 2}\left(x_{i}\right)$. Note that we throw out the multiplier $\sqrt{2 L}$ ! in the upper bound since it is bounded by a constant that only depends on $n$ when $L \leq 2 n^{3}$. Thus

$$
\left|\mathcal{I}_{\vec{m}}\right|=C^{L} \int_{0}^{\infty} e^{t(\alpha-1) \sigma} \prod_{i=1}^{L}\left(\int_{\mathbb{R}} F_{\frac{2}{3}, 2}(x)\left|\phi_{e^{-t \sigma, t}}^{\left(m_{i}\right)}(x)\right| d x\right) d \sigma
$$

Applying Proposition 5.2 (c), (d). For each $\int_{\mathbb{R}} F_{\frac{2}{3}, 2}(x)\left|\phi_{e^{-t \sigma, t}}^{\left(m_{i}\right)}(x)\right| d x, i=1, \ldots, r$, since $m_{i} \in \mathbb{Z}_{\geq 1}$, this integral can be upper bounded by $C t^{\frac{1}{6}} \exp \left(t U_{m_{i}}\left(\sigma \wedge m_{i}^{2}\right)\right)$. When $i \geq r+1, m_{i}=0$, the integral can be upper bounded by $C t^{\frac{1}{6}} \exp \left(t U_{1}(\sigma \wedge 1)-t \sigma\right)$. Therefore, there exists a constant $C=C\left(n, t_{0}\right)$ such that for all $t>t_{0}, 2 \leq L \leq 2 n^{3}$ and $\vec{m} \in \mathfrak{M}(L, n)$,

$$
\left|\mathcal{I}_{\vec{m}}\right| \leq C^{L} t^{\frac{L}{6}} \int_{0}^{\infty} e^{t \mathrm{M}_{2}(\sigma)} d \sigma
$$

where $\mathrm{M}_{2}(\sigma)=(\alpha-1) \sigma+\sum_{i=1}^{r} U_{m_{i}}\left(\sigma \wedge m_{i}^{2}\right)+(L-r)\left(U_{1}(\sigma \wedge 1)-\sigma\right)$. To conclude the proof of Proposition 5.3 , it suffices to show that there exists $C=C\left(n, t_{0}\right)$ such that for all $t>t_{0}$ and $L \geq 2$ and $\vec{m} \in \mathfrak{M}(L, n)$,

$$
\int_{0}^{\infty} e^{t \mathrm{M}_{2}(\sigma)} d \sigma \leq C e^{\frac{p^{3}}{3} t-\delta_{p} t}
$$

Once this is shown, applying (5.11) completes the proof of the Proposition 5.3.

We are left to show (5.12). To this aim, we divide our argument into two cases, depending on $r=1$ or not.

Case 1. $r=1$. In this case, $m_{1}=n$ and $m_{i}=0$ for $i>1$. As a result,

$$
\mathrm{M}_{2}(\sigma)=(\alpha-1) \sigma+U_{n}\left(\sigma \wedge n^{2}\right)+(L-1)\left(U_{1}(\sigma \wedge 1)-\sigma\right) .
$$


We decompose

$$
\int_{0}^{\infty} e^{t \mathrm{M}_{2}(\sigma)} d \sigma=\left(\int_{0}^{1}+\int_{1}^{n}+\int_{n}^{\infty}\right) e^{t \mathrm{M}_{2}(\sigma)} d \sigma=\mathcal{I}_{1}+\mathcal{I}_{2}+\mathcal{I}_{3}
$$

and we are going to upper bound $\mathcal{I}_{1}, \mathcal{I}_{2}, \mathcal{I}_{3}$ respectively.

For $\mathcal{I}_{1}$, when $\sigma \leq 1$, the right hand side of (5.13) can be simplified as

$$
\mathrm{M}_{2}(\sigma)=(\alpha-L) \sigma+U_{n}(\sigma)+(L-1) U_{1}(\sigma)=p \sigma-\frac{2 L}{3} \sigma^{\frac{3}{2}} .
$$

Since $L \geq 2$, similar to the discussion in Stage $1, \mathrm{M}_{2}(\sigma)=p \sigma-\frac{2 L}{3} \sigma^{\frac{3}{2}} \leq p \sigma-\frac{4}{3} \sigma^{\frac{3}{2}} \leq \frac{p^{3}}{12}$. Thereby,

$$
\mathcal{I}_{1}=\int_{0}^{1} e^{t \mathrm{M}_{2}(\sigma)} d \sigma \leq \int_{0}^{1} e^{\frac{p^{3} t}{12}} d \sigma=e^{\frac{p^{3} t}{12}} .
$$

For $\mathcal{I}_{2}$, when $1 \leq \sigma \leq n^{2}$, We can simplify $\mathrm{M}_{2}(\sigma)=p \sigma-\frac{2}{3} \sigma^{\frac{3}{2}}+(L-1)\left(\frac{1}{3}-\sigma\right)$. Note that the maximum of $p \sigma-\frac{2}{3} \sigma^{\frac{3}{2}}$ under the condition $\sigma \geq 0$ equals $\frac{1}{3} p^{3}$. Using this in conjunction with $L \geq 2, \mathrm{M}_{2}(\sigma) \leq \frac{1}{3} p^{3}+\frac{1}{3}-\sigma$. As a result,

$$
\mathcal{I}_{2}=\int_{1}^{n^{2}} e^{t \mathrm{M}_{2}(\sigma)} d \sigma \leq e^{\frac{p^{3}}{3} t} \int_{1}^{\infty} e^{\left(\frac{1}{3}-\sigma\right) t} d \sigma=t^{-1} e^{\frac{p^{3}-2}{3} t} .
$$

For $\mathcal{I}_{3}$, the right hand side of (5.13) simplifies to

$$
\mathrm{M}_{2}(\sigma)=(\alpha-1) \sigma+U_{n}\left(n^{2}\right)+(L-1)\left(U_{1}(1)-\sigma\right)=(\alpha-1) \sigma+\frac{1}{3} n^{3}+(L-1)\left(\frac{1}{3}-\sigma\right) .
$$

Since $\alpha<1, \sigma \geq n^{2}$ and $L \geq 2$,

$$
\mathrm{M}_{2}(\sigma) \leq n^{2}(\alpha-1)+\frac{1}{3} n^{3}+(L-1)\left(\frac{1}{3}-\sigma\right) \leq n^{2}(\alpha-1)+\frac{1}{3} n^{3}+\left(\frac{1}{3}-\sigma\right)
$$

Note that $n^{2}(\alpha-1)+\frac{1}{3} n^{3} \leq \frac{1}{3}(n+\alpha-1)^{3}=\frac{1}{3} p^{3}$, hence $\mathrm{M}_{1}(\sigma) \leq \frac{1}{3} p^{3}+\frac{1}{3}-\sigma$. Thereby,

$$
\int_{n^{2}}^{\infty} e^{t \mathrm{M}_{2}(\sigma)} d \sigma \leq e^{\frac{p^{3} t}{3}} \int_{n^{2}}^{\infty} e^{\left(\frac{1}{3}-\sigma\right) t} d \sigma=t^{-1} e^{\left(\frac{p^{3}+1}{3}-n^{2}\right) t} \leq t^{-1} e^{\frac{p^{3}-2}{3} t} .
$$

Combining (5.14), (5.15) and (5.16), we conclude that for $t \geq t_{0}$,

$$
\int_{0}^{\infty} e^{t \mathrm{M}_{2}(\sigma)} d \sigma \leq\left(e^{\frac{1}{12} p^{3} t}+t^{-1} e^{\frac{p^{3}-2}{3} t}+t^{-1} e^{\frac{p^{3}-2}{3} t}\right) \leq C e^{\frac{1}{3} p^{3} t-\delta_{p} t}
$$

The last inequality follows since $\delta_{p}=\min \left(\frac{2}{3}, \frac{1}{4} p^{3}\right)$. So far we have shown (5.12) when $r=1$.

Case 2. $r \geq 2$. This implies $n \geq 2$. We write

$$
\int_{0}^{\infty} e^{t \mathrm{M}_{2}(\sigma)} d \sigma=\left(\int_{0}^{1}+\int_{1}^{\infty}\right) e^{t \mathrm{M}_{2}(\sigma)} d \sigma=\widetilde{\mathcal{I}}_{1}+\widetilde{\mathcal{I}}_{2}
$$

For $\widetilde{\mathcal{I}}_{1}$, when $\sigma \leq 1, \mathrm{M}_{2}(\sigma)=p \sigma-\frac{2}{3} L \sigma^{3}$. Via the same argument as in Case 1, we conclude that $\widetilde{\mathcal{I}}_{1} \leq e^{\frac{1}{12} p^{3} t}$. For $\widetilde{\mathcal{I}}_{2}$, using the inequality $U_{m_{i}}\left(\sigma \wedge m_{i}^{2}\right) \leq \frac{1}{3} m_{i}^{3}$ and $U_{1}(\sigma \wedge 1)=U_{1}(1)=\frac{1}{3}$, we get

$$
\mathrm{M}_{2}(\sigma) \leq \frac{1}{3} \sum_{i=1}^{r} m_{i}^{3}+\frac{1}{3}(L-r)+(\alpha-L+r-1) \sigma \leq \frac{1}{3} \sum_{i=1}^{r} m_{i}^{3}+(\alpha-1) \sigma
$$

Since we assume $r \geq 2$, it is convincible that $\sum_{i=1}^{r} m_{i}^{3}$ is at most $(n-1)^{3}+1$, since the cubic sum will increase if we let mass concentrate on fewer terms. To justify this, note that $\sum_{i=2}^{r} m_{i}^{3} \leq\left(\sum_{i=2}^{r} m_{i}\right)^{3}=\left(n-m_{1}\right)^{3}$. Thus

$$
\sum_{i=1}^{r} m_{i}^{3} \leq m_{1}^{3}+\left(n-m_{1}\right)^{3}=(n-1)^{3}+1+3 n\left(m_{1}-1\right)\left(m_{1}-(n-1)\right) \leq(n-1)^{3}+1 .
$$

Applying this inequality to the right hand side of (5.17), we see that

$$
\mathrm{M}_{2}(\sigma) \leq \frac{1}{3}\left((n-1)^{3}+1\right)+(\alpha-1) \sigma \leq \frac{1}{3}(n-1)^{3}+\alpha-\frac{2}{3} \leq \frac{1}{3}(\alpha+n-1)^{3}-\frac{2}{3} .
$$


The second inequality above follows from $\sigma \geq 1$ and the third equality is due to $\frac{1}{3}(n-1)^{3}+\alpha \leq \frac{1}{3}(n-1)^{3}+$ $(n-1)^{2} \alpha \leq \frac{1}{3}(n-1+\alpha)^{3}$. Recall that $p=\alpha+n-1$, we obtain $\mathrm{M}_{2}(\sigma) \leq \frac{1}{3} p^{3}-\frac{2}{3}+(\alpha-1) \sigma$, and thus $\widetilde{\mathcal{I}}_{2} \leq \int_{1}^{\infty} \exp \left(t\left(\frac{1}{3} p^{3}-\frac{2}{3}+(\alpha-1) \sigma\right)\right) d \sigma=\frac{1}{1-\alpha} e^{\left(\frac{1}{3} p^{3}-\frac{2}{3}\right) t}$. So there exists a constant $C$ such that for $t>t_{0}$,

$$
\int_{0}^{\infty} e^{t \mathrm{M}_{2}(\sigma)} d \sigma=\widetilde{\mathcal{I}}_{1}+\widetilde{\mathcal{I}}_{2} \leq e^{\frac{p^{3} t}{12}}+(1-\alpha)^{-1} e^{\frac{p^{3}-2}{3} t} \leq C e^{\frac{p^{3}}{3} t-\delta_{p} t} .
$$

This implies (5.12) and completes the proof of Stage 2.

Proof of Proposition 2.9. It suffices to prove that for fixed $p>0$, there exists a constant $C=C(p)$ such that for all $L \geq 2$ and $t>1$,

$$
\left|\mathcal{B}_{p, L}(t)\right| \leq \frac{C^{L}(2 L)^{\frac{L}{2}}}{L !} t^{\frac{L}{6}} e^{\frac{p^{3}}{3} t-\delta_{p} t} .
$$

One this is shown, we conclude our proof by observing

$$
\left|\sum_{L=2}^{\infty} \mathcal{B}_{p, L}(t)\right| \leq \sum_{L=2}^{\infty}\left|\mathcal{B}_{p, L}(t)\right| \leq e^{\frac{p^{3}}{3} t-\delta_{p} t} \sum_{L=2}^{\infty} \frac{C^{L}(2 L)^{\frac{L}{2}} t^{\frac{L}{6}}}{L !}
$$

Using the inequality of Stirling's formula, ew know that $L^{L} \leq e^{L} L$ ! for all $L \in \mathbb{Z}_{\geq 1}$. Consequently, $(2 L)^{\frac{L}{2}}=$ $2^{L}\left(L^{L}\right)^{\frac{1}{2}} \leq 2^{L} e^{\frac{L}{2}} \sqrt{L !}$. So there exists constant $C_{1}, C_{2}$ such that for all $t>1$,

$$
\sum_{L=2}^{\infty} \frac{C^{L}(2 L)^{\frac{L}{2}} t^{\frac{L}{6}}}{L !} \leq \sum_{L=2}^{\infty} \frac{C_{1}^{L} t^{\frac{L}{6}}}{\sqrt{L !}} \leq e^{C_{2} t^{\frac{1}{3}}}
$$

Combining the above inequality with (5.19), the left hand side of (5.19) is upper bounded by $\exp \left(p^{3} t / 3-\right.$ $\left.\delta_{p} t+C_{2} t^{\frac{1}{3}}\right)$. Taking the logarithm and dividing by $t$ for both sides and letting $t \rightarrow \infty$ completes the proof of Proposition 2.9 .

We are left to show (5.18). referring to (2.12) and using Leibniz's rule,

$$
\mathcal{B}_{p, L}(t)=\frac{(-1)^{n}}{\Gamma(1-\alpha)} \sum_{\vec{m} \in \mathfrak{M}(L, n)} \int_{0}^{1} s^{-\alpha} \frac{1}{L !} \int_{\mathbb{R}^{L}} \operatorname{Pf}\left[K\left(x_{i}, x_{j}\right)\right]_{i, j=1}^{L} \prod_{i=1}^{L} \phi_{s, t}^{\left(m_{i}\right)}\left(x_{i}\right) d x=\frac{(-1)^{n}}{\Gamma(1-\alpha) L !} \sum_{\vec{m} \in \mathfrak{M}(L, n)}\left(\begin{array}{c}
n \\
\vec{m}
\end{array}\right) \mathcal{I}_{\vec{m}}
$$

where $\mathcal{I}_{\vec{m}}$ is defined in (5.7). Using Proposition 5.3 and the above display, recalling that $\# \mathfrak{M}(L, n)$ represents the number of elements that lie in $\mathfrak{M}(L, n)$, we get for all $L \geq 2$,

$$
\left|\mathcal{B}_{p, L}(t)\right| \leq \frac{1}{\Gamma(1-\alpha) L !} \sum_{\vec{m} \in \mathfrak{M}(L, n)}\left(\begin{array}{l}
n \\
\vec{m}
\end{array}\right)\left|\mathcal{I}_{\vec{m}}\right| \leq \frac{n !}{\Gamma(1-\alpha)}(\# \mathfrak{M}(L, n)) \max _{\vec{m} \in \mathfrak{M}(L, n)}\left|\mathcal{I}_{\vec{m}}\right|
$$

where the first inequality follows from taking the absolute value of both sides of (5.20) and applying triangle inequality to the right hand side. The second inequality follows from upper bounding $\left(\begin{array}{l}n ! \\ \vec{m}\end{array}\right)$ with $n$ !. Recall from (4.7) that $\# \mathfrak{M}(L, n) \leq L^{n}$. To prove (5.18), applying Proposition 5.3 to upper bound each $\left|\mathcal{I}_{\vec{m}}\right|$, we obtain

$$
\left|\mathcal{B}_{p, L}(t)\right| \leq \frac{n ! L^{n}}{\Gamma(1-\alpha) L !} C^{L}(2 L)^{\frac{L}{6}} t^{\frac{L}{6}} e^{\frac{p^{3} t}{3}-\delta_{p} t} .
$$

Note that $L^{n}$ grows slower than $C_{1}^{L}$ for $C_{1}>1$, as $L \rightarrow \infty$. So there exists a constant $C_{1}$ such that $\frac{n ! L^{n}}{\Gamma(1-\alpha)} C^{L} \leq$ $C_{1}^{L}$. Applying this inequality to the right hand side of the above display completes the proof of (5.18).

\section{Appendix A. Basic faCts of Airy function}

In this section, we review some basic properties of the Airy function.

Lemma A.1. We have the following asymptotics for Airy function

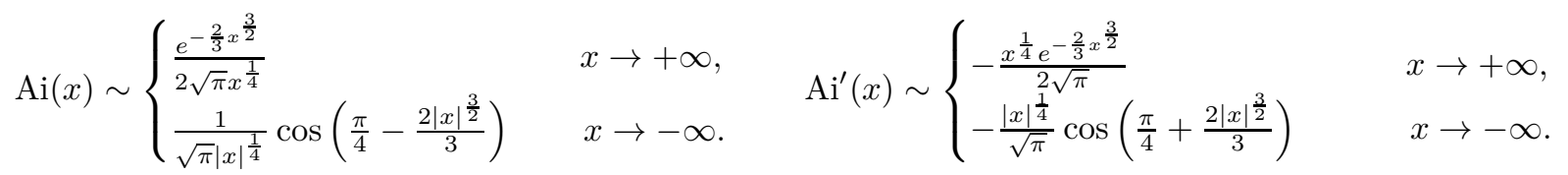

Proof. See Eq 10.4.59-10.4.62 of [AS48].

Lemma A.2. We have $\int_{-\infty}^{\infty} \operatorname{Ai}(x) d x=1$. and $\int_{-\infty}^{0} \operatorname{Ai}(x) d x=1 / 3$. 
Proof. See page 431 of [Olv97].

Lemma A.3. There exists constant $C$ such that

$$
\begin{array}{ll}
\frac{1}{C(x+1)} e^{-\frac{4}{3} x^{\frac{3}{2}}} \leq \int_{0}^{\infty} \operatorname{Ai}(x+\lambda)^{2} d \lambda \leq \frac{C}{x+1} e^{-\frac{4}{3} x^{\frac{3}{2}}} & \forall x \geq 0 \\
\frac{1}{C}(\sqrt{|x|}+1) \leq \int_{0}^{\infty} \operatorname{Ai}(x+\lambda)^{2} d \lambda \leq C(\sqrt{|x|}+1), & \forall x \leq 0
\end{array}
$$

Proof. This is Eq 2.8 and Eq 2.9 of [DT19].

\section{Appendix B. Estimate of the Pfaffian Kernel}

In this section, we provide various bounds for the entries in the GOE Pfaffian kernel. As a notational convention, we say $f(x) \sim g(x)$ as $x \rightarrow a$ (where $a$ can be $\pm \infty$ ) if $\lim _{x \rightarrow a} \frac{f(x)}{g(x)}=1$.

Lemma B.1. There exists a constant $C>0$ such that

$\begin{array}{ll}\text { (i) } \frac{\exp \left(-\frac{2}{3} x^{\frac{3}{2}}\right)}{C(1+x)^{\frac{1}{4}}} \leq K_{12}(x, x) \leq \frac{C \exp \left(-\frac{2}{3} x^{\frac{3}{2}}\right)}{(1+x)^{\frac{1}{4}}} & \forall x \geq 0, \\ \text { (ii) } 0 \leq K_{12}(x, x) \leq C \sqrt{1-x} & \forall x \leq 0 .\end{array}$

Proof. We first prove (i). By setting $x=y$ in (2.2), we get

$$
K_{12}(x, x)=\int_{0}^{\infty} \operatorname{Ai}(x+\lambda)^{2} d \lambda+\frac{1}{2} \operatorname{Ai}(x) \int_{-\infty}^{x} \operatorname{Ai}(\lambda) d \lambda .
$$

For the second term in the above display, by Lemma A.1 and Lemma A.2, we have as $x \rightarrow+\infty$

$$
\operatorname{Ai}(x) \int_{-\infty}^{x} \operatorname{Ai}(\lambda) d \lambda \sim \frac{e^{-\frac{2}{3} x^{\frac{3}{2}}}}{2 \sqrt{\pi} x^{\frac{1}{4}}}
$$

Combining this with the first inequality of Lemma A.3, which controls the first term on the right hand side, the upper bound in (i) naturally follows. To prove the lower bound of (i), due to the above displayed asymptotic and the non-negativity of $\int_{0}^{\infty} \operatorname{Ai}(x+\lambda)^{2} d \lambda$, there exists constant $M$ and $C$ such that for $x>M$,

$$
K_{12}(x, x) \geq C^{-1} x^{-\frac{1}{4}} \exp \left(-\frac{2}{3} x^{\frac{3}{2}}\right) .
$$

To conclude the lower bound in (i), it suffices to show that the minimum of $K_{12}(x, x)$ is positive over [0,M] $\left(K_{12}(x, x)\right.$ is continuous, so admits a minimum). Due to Eq. (B.1) and Lemma A.2, we can rewrite $K_{12}(x, x)=$ $\int_{0}^{\infty} \operatorname{Ai}(x+\lambda)^{2} d \lambda+\frac{1}{3} \operatorname{Ai}(x)+\frac{1}{2} \operatorname{Ai}(x) \int_{0}^{x} \operatorname{Ai}(\lambda) d \lambda$. Since $\operatorname{Ai}(x)$ is positive for $x \geq 0$, this implies $K_{12}(x, x)>0$ for all $x>0$, which completes the proof of the lower bound.

We move on proving (ii). The lower bound follows directly since $K_{12}(x, x)$ is the first order correlation function of a Pfaffian point process, thus is negative. For the upper bound, by the asymptotic of $\operatorname{Ai}(x)$ at $-\infty$, there exists constant $C$ such that for all $x \leq 0$,

$$
\left|\operatorname{Ai}(x) \int_{-\infty}^{x} \operatorname{Ai}(\lambda) d \lambda\right| \leq C(1+|x|)^{-\frac{1}{4}}
$$

The result then follows from the second inequality of Lemma A.3 and (B.1).

Recall that we defined $F_{\alpha, \beta}(x)=C\left(e^{-\alpha x^{\frac{3}{2}}} \mathbf{1}_{\{x \geq 0\}}+(1-x)^{\beta} \mathbf{1}_{\{x<0\}}\right)$.

Lemma B.2. There exists a constant $C$, such that for all $x, y \in \mathbb{R}$, we have the following upper bounds for the Pfaffian kernel entries:

(a) $\left|K_{11}(x, y)\right| \leq C\left(F_{\frac{2}{3}, \frac{5}{4}}(x) \wedge F_{\frac{2}{3}, \frac{3}{4}}(x) F_{\frac{2}{3}, \frac{3}{4}}(y)\right)$

(b) $\left|K_{12}(x, y)\right| \leq C\left(F_{\frac{2}{3}, \frac{3}{4}}(x) \wedge F_{0, \frac{3}{4}}(y)\right)$

(c) $\left|K_{22}(x, y)\right| \leq C F_{0, \frac{3}{4}}(x)$ 
Proof. For (a), it suffices to show that $\left|K_{11}(x, y)\right| \leq C F_{\frac{2}{3}, \frac{5}{4}}(x)$ and $\left|K_{11}(x, y)\right| \leq C F_{\frac{2}{3}, \frac{3}{4}}(x) F_{\frac{2}{3}, \frac{3}{4}}(y)$. Recall the expression of $K_{11}(x, y)$ from (2.1). Using integration by parts for the right hand side of (2.1), we get $K_{11}(x, y)=\operatorname{Ai}(x) \operatorname{Ai}(y)-2 \int_{0}^{\infty} \operatorname{Ai}(y+\lambda) \operatorname{Ai}^{\prime}(x+\lambda) d \lambda$. This implies that $\left|K_{11}(x, y)\right| \leq|\operatorname{Ai}(x) \operatorname{Ai}(y)|+2 \int_{0}^{\infty} \mid \operatorname{Ai}(y+$ $\lambda) \operatorname{Ai}^{\prime}(x+\lambda) \mid d \lambda$. Since $|\operatorname{Ai}(x)|$ is a bounded function, there exists constant $C$ such that

$$
\left|K_{11}(x, y)\right| \leq C|\operatorname{Ai}(x)|+C \int_{0}^{\infty}\left|\operatorname{Ai}^{\prime}(x+\lambda)\right| d \lambda=C+C \int_{x}^{\infty}\left|\operatorname{Ai}^{\prime}(\lambda)\right| d \lambda .
$$

To obtain the upper bound for $|\operatorname{Ai}(x)|$ and $\int_{x}^{\infty}|\operatorname{Ai}(\lambda)| d \lambda$, it suffices to look at their behavior as $x \rightarrow \pm \infty$. The asymptotic $\operatorname{Ai}^{\prime}(x)$ at $\pm \infty$ is specified in Lemma A.1. Therefore,

$$
\int_{x}^{\infty}\left|\operatorname{Ai}^{\prime}(\lambda)\right| d \lambda \leq C e^{-\frac{2}{3} x^{\frac{3}{2}}}, \text { if } x \geq 0 ; \quad \int_{x}^{\infty}\left|\operatorname{Ai}^{\prime}(\lambda)\right| d \lambda \leq C(1-x)^{\frac{5}{4}} \text { if } x \leq 0 .
$$

This implies that $\left|K_{11}(x, y)\right| \leq C F_{\frac{2}{3}, \frac{5}{4}}(x)$. In addition, since

$$
K_{11}(x, y)=\int_{0}^{\infty} \operatorname{Ai}(x+\lambda) \operatorname{Ai}^{\prime}(y+\lambda) d \lambda-\int_{0}^{\infty} \operatorname{Ai}^{\prime}(x+\lambda) \operatorname{Ai}(y+\lambda) d \lambda=A_{1}-A_{2}
$$

By Cauchy Schwartz inequality,

$$
A_{1}^{2} \leq \int_{0}^{\infty} \operatorname{Ai}(x+\lambda)^{2} d \lambda \int_{0}^{\infty} \operatorname{Ai}^{\prime}(y+\lambda)^{2} d \lambda=\int_{x}^{\infty} \operatorname{Ai}(\lambda)^{2} d \lambda \int_{y}^{\infty} \operatorname{Ai}^{\prime}(\lambda)^{2} d \lambda .
$$

By Lemma A.1, $\operatorname{Ai}(x)^{2}$ decays asymptotically as $\exp \left(-\frac{4}{3} x^{\frac{3}{2}}\right)$ as $x \rightarrow+\infty$ and is asymptotically upper bounded by $|x|^{-\frac{1}{2}}$ as $x \rightarrow-\infty$. This implies that $\int_{x}^{\infty} \operatorname{Ai}(\lambda)^{2} d \lambda \leq C F_{\frac{4}{3}, \frac{1}{2}}(x)$. Similarly, $\operatorname{Ai}^{\prime}(y)^{2}$ decays asymptotically as $\exp \left(-\frac{4}{3} y^{\frac{3}{2}}\right)$ and is asymptotically upper bounded by $|y|^{\frac{1}{2}}$, we get $\int_{y}^{\infty} \operatorname{Ai}^{\prime}(\lambda)^{2} d \lambda \leq C F_{\frac{4}{3}, \frac{3}{2}}(y)$. As a result,

$$
\left|A_{1}\right| \leq\left(\int_{x}^{\infty} \operatorname{Ai}(\lambda)^{2} d \lambda\right)^{\frac{1}{2}}\left(\int_{y}^{\infty} \operatorname{Ai}^{\prime}(\lambda)^{2} d \lambda\right)^{\frac{1}{2}} \leq C F_{\frac{2}{3}, \frac{1}{4}}(x) F_{\frac{2}{3}, \frac{3}{4}}(y) \leq C F_{\frac{2}{3}, \frac{3}{4}}(x) F_{\frac{2}{3}, \frac{3}{4}}(y) .
$$

For the second inequality above, we use the property that $\sqrt{F_{\alpha, \beta}}=F_{\alpha / 2, \beta / 2}$ and for the third inequality, $F_{\alpha, \beta}(x)$ is increasing in $\beta$. Interchanging the role of $x$ and $y$, we also have $\left|A_{2}\right| \leq C F_{\frac{2}{3}, \frac{3}{4}}(x) F_{\frac{2}{3}, \frac{3}{4}}(y)$. Therefore, the same upper bound holds for $\left|K_{11}(x, y)\right|$ and we conclude the proof of (a).

We move on showing (b). We will prove $\left|K_{12}(x, y)\right| \leq C F_{\frac{2}{3}, \frac{3}{4}}(x)$ and $\left|K_{12}(x, y)\right| \leq C F_{0, \frac{3}{4}}(y)$ respectively. Recall $K_{12}(x, y)$ from $(2.2)$. Note that both $|\operatorname{Ai}(y+\lambda)|$ and $\left|\int_{-\infty}^{y} \operatorname{Ai}(\lambda) d \lambda\right|$ are bounded function of $y$ (see Lemma A.2), by using triangle inequality,

$$
\left|K_{12}(x, y)\right| \leq \frac{1}{2} \int_{0}^{\infty}|\operatorname{Ai}(x+\lambda) \operatorname{Ai}(y+\lambda)| d \lambda+\frac{1}{2}|\operatorname{Ai}(x)| \cdot\left|\int_{-\infty}^{y} \operatorname{Ai}(\lambda) d \lambda\right| \leq C \int_{x}^{\infty}|\operatorname{Ai}(\lambda)| d \lambda+C|\operatorname{Ai}(x)| .
$$

By the asymptotic of $\operatorname{Ai}(x)$ at $\pm \infty$, (use the similar approach as in part (a)), we see that $\left|K_{12}(x, y)\right| \leq C F_{\frac{2}{3}, \frac{3}{4}}(x)$. We proceed to obtain a different upper bound for $K_{12}$. Referring to the right hand side of the first inequality in the above display and upper bounding $|\operatorname{Ai}(x+\lambda)|$ and $\left|\frac{1}{2} \operatorname{Ai}(x) \int_{-\infty}^{y} \operatorname{Ai}(\lambda) d \lambda\right|$ by a constant, we find that

$$
\left|K_{12}(x, y)\right| \leq C \int_{0}^{\infty}|\operatorname{Ai}(y+\lambda)| d \lambda+C \leq C F_{0, \frac{3}{4}}(y) .
$$

This concludes our proof of (b).

Finally, let us demonstrate (c). Recall from (2.3) that

$$
\begin{aligned}
K_{22}(x, y)= & \frac{1}{4} \int_{0}^{\infty} \operatorname{Ai}(x+\lambda)\left(\int_{\lambda}^{\infty} \operatorname{Ai}(y+\mu) d \mu\right) d \lambda-\frac{1}{4} \int_{0}^{\infty} \operatorname{Ai}(y+\lambda)\left(\int_{\lambda}^{\infty} \operatorname{Ai}(x+\mu) d \mu\right) d \lambda \\
& -\frac{1}{4} \int_{0}^{\infty} \operatorname{Ai}(x+\lambda) d \lambda+\frac{1}{4} \int_{0}^{\infty} \operatorname{Ai}(y+\lambda) d \lambda-\frac{\operatorname{sgn}(x-y)}{4}
\end{aligned}
$$

and recall that sgn is the sign function. By Fubini's theorem,

$$
\int_{0}^{\infty} \operatorname{Ai}(y+\lambda)\left(\int_{\lambda}^{\infty} \operatorname{Ai}(x+\mu) d \mu\right) d \lambda=\left(\int_{0}^{\infty} \operatorname{Ai}(x+\lambda) d \lambda\right)\left(\int_{0}^{\infty} \operatorname{Ai}(y+\lambda) d \lambda\right)-\int_{0}^{\infty} \operatorname{Ai}(x+\lambda)\left(\int_{\lambda}^{\infty} \operatorname{Ai}(y+\mu) d \mu\right) d \lambda
$$


Replacing the term $\int_{0}^{\infty} \operatorname{Ai}(y+\lambda)\left(\int_{\lambda}^{\infty} \operatorname{Ai}(x+\mu) d \mu\right) d \lambda$ in (B.2) with the right hand side in the above display,

$$
\begin{aligned}
K_{22}(x, y)= & \frac{1}{2} \int_{0}^{\infty} \operatorname{Ai}(x+\lambda)\left(\int_{\lambda}^{\infty} \operatorname{Ai}(y+\mu) d \mu\right) d \lambda-\frac{1}{4}\left(\int_{0}^{\infty} \operatorname{Ai}(x+\lambda) d \lambda\right)\left(\int_{0}^{\infty} \operatorname{Ai}(y+\lambda) d \lambda\right) \\
& -\frac{1}{4} \int_{0}^{\infty} \operatorname{Ai}(x+\lambda) d \lambda+\frac{1}{4} \int_{0}^{\infty} \operatorname{Ai}(y+\lambda) d \lambda-\frac{\operatorname{sgn}(x-y)}{4} .
\end{aligned}
$$

We know that $\left|\int_{0}^{\infty} \operatorname{Ai}(x+\lambda) d \lambda\right|,\left|\int_{0}^{\infty} \operatorname{Ai}(y+\lambda) d \lambda\right|$ can upper bounded by a constant. Applying triangle inequality to the above display,

$$
\left|K_{22}(x, y)\right| \leq C \int_{0}^{\infty}|\operatorname{Ai}(x+\lambda)| d \lambda+C .
$$

Using the asymptotic of $\mathrm{Ai}(x)$ at $\pm \infty$ in Lemma A.1, we find that $\left|K_{22}(x, y)\right| \leq C F_{0, \frac{3}{4}}(x)$, thus conclude (c).

\section{REFERENCES}

[ACQ11] Gideon Amir, Ivan Corwin, and Jeremy Quastel. Probability distribution of the free energy of the continuum directed random polymer in 1+ 1 dimensions. Communications on pure and applied mathematics, 64(4):466-537, 2011.

[AGZ10] Greg W Anderson, Alice Guionnet, and Ofer Zeitouni. An introduction to random matrices, volume 118. Cambridge university press, 2010.

[AS48] Milton Abramowitz and Irene A Stegun. Handbook of mathematical functions with formulas, graphs, and mathematical tables, volume 55. US Government printing office, 1948.

[BBC16] Alexei Borodin, Alexey Bufetov, and Ivan Corwin. Directed random polymers via nested contour integrals. Annals of Physics, 368:191-247, 2016.

[BBC20] Guillaume Barraquand, Alexei Borodin, and Ivan Corwin. Half-space Macdonald processes. In Forum of Mathematics, $P i$, volume 8. Cambridge University Press, 2020.

[BBCS18] Jinho Baik, Guillaume Barraquand, Ivan Corwin, and Toufic Suidan. Pfaffian Schur processes and last passage percolation in a half-quadrant. The Annals of Probability, 46(6):3015-3089, 2018.

[BBCW18] Guillaume Barraquand, Alexei Borodin, Ivan Corwin, and Michael Wheeler. Stochastic six-vertex model in a halfquadrant and half-line open asymmetric simple exclusion process. Duke Mathematical Journal, 167(13):2457-2529, 2018.

[BC95] Lorenzo Bertini and Nicoletta Cancrini. The stochastic heat equation: Feynman-Kac formula and intermittence. Journal of statistical Physics, 78(5-6):1377-1401, 1995.

[BC14] Alexei Borodin and Ivan Corwin. Macdonald processes. Probability Theory and Related Fields, 158(1-2):225-400, 2014.

[BC16] Raluca M Balan and Daniel Conus. Intermittency for the wave and heat equations with fractional noise in time. The Annals of Probability, 44(2):1488-1534, 2016.

[BFO20] Dan Betea, Patrik L Ferrari, and Alessandra Occelli. Stationary half-space last passage percolation. Communications in Mathematical Physics, pages 1-47, 2020.

[BG16] Alexei Borodin and Vadim Gorin. Moments match between the kpz equation and the airy point process. SIGMA. Symmetry, Integrability and Geometry: Methods and Applications, 12:102, 2016.

[BGS17] Riddhipratim Basu, Shirshendu Ganguly, and Allan Sly. Upper tail large deviations in first passage percolation. arXiv preprint arXiv:1712.01255, 2017.

[BKD20] Guillaume Barraquand, Alexandre Krajenbrink, and Pierre Le Doussal. Half-space stationary kardar-parisi-zhang equation. arXiv preprint arXiv:2003.03809, 2020.

[BR01] Jinho Baik and Eric M Rains. The asymptotics of monotone subsequences of involutions. Duke Mathematical Journal, 109(2):205-281, 2001.

[CC19] Mattia Cafasso and Tom Claeys. A Riemann-Hilbert approach to the lower tail of the KPZ equation. arXiv preprint arXiv:1910.02493, 2019.

[CCM94] René Carmona, René A Carmona, and Stanislav A Molchanov. Parabolic Anderson problem and intermittency, volume 518. American Mathematical Soc., 1994.

[CD15] Le Chen and Robert C Dalang. Moments and growth indices for the nonlinear stochastic heat equation with rough initial conditions. The Annals of Probability, 43(6):3006-3051, 2015.

[CG20a] Ivan Corwin and Promit Ghosal. KPZ equation tails for general initial data. Electronic Journal of Probability, 25, 2020.

[CG20b] Ivan Corwin and Promit Ghosal. Lower tail of the KPZ equation. Duke Mathematical Journal, 169(7):1329-1395, 2020.

[Che15] Xia Chen. Precise intermittency for the parabolic Anderson equation with an $(1+1)$-dimensional time-space white noise. Annales de l'IHP Probabilités et statistiques, 51(4):1486-1499, 2015.

[CJK13] Daniel Conus, Mathew Joseph, and Davar Khoshnevisan. On the chaotic character of the stochastic heat equation, before the onset of intermittency. The Annals of Probability, 41(3B):2225-2260, 2013.

[CJKS13] Daniel Conus, Mathew Joseph, Davar Khoshnevisan, and Shang-Yuan Shiu. On the chaotic character of the stochastic heat equation, II. Probability Theory and Related Fields, 156(3-4):483-533, 2013.

[Cor12] Ivan Corwin. The Kardar-Parisi-Zhang equation and universality class. Random matrices: Theory and applications, 1(01):1130001, 2012.

[CS18] Ivan Corwin and Hao Shen. Open ASEP in the weakly asymmetric regime. Communications on Pure and Applied Mathematics, 71(10):2065-2128, 2018. 
[DNKDT19] Jacopo De Nardis, Alexandre Krajenbrink, Pierre Le Doussal, and Thimothée Thiery. Delta-Bose gas on a half-line and the KPZ equation: boundary bound states and unbinding transitions. arXiv preprint arXiv:1911.06133, 2019.

[DT19] Sayan Das and Li-Cheng Tsai. Fractional moments of the Stochastic Heat Equation. arXiv preprint arXiv:1910.09271, 2019.

[Fer04] Patrik L Ferrari. Polynuclear growth on a flat substrate and edge scaling of GOE eigenvalues. Communications in mathematical physics, 252(1-3):77-109, 2004.

[FK09] Mohammud Foondun and Davar Khoshnevisan. Intermittence and nonlinear parabolic stochastic partial differential equations. Electronic Journal of Probability, 14:548-568, 2009.

[GH19] Máté Gerencsér and Martin Hairer. Singular SPDEs in domains with boundaries. Probability Theory and Related Fields, 173(3-4):697-758, 2019.

[Gho18] Promit Ghosal. Moments of the SHE under delta initial measure. arXiv preprint arXiv:1808.04353, 2018.

[GKM07] Jürgen Gärtner, Wolfgang König, and Stanislav Molchanov. Geometric characterization of intermittency in the parabolic Anderson model. The Annals of Probability, 35(2):439-499, 2007.

[GL20] Promit Ghosal and Yier Lin. Lyapunov exponents of the SHE for general initial data. arXiv preprint arXiv:200\%.06505, 2020 .

[GLD12] Thomas Gueudré and Pierre Le Doussal. Directed polymer near a hard wall and KPZ equation in the half-space. EPL (Europhysics Letters), 100(2):26006, 2012.

[GM90] Jürgen Gärtner and Stanislav A Molchanov. Parabolic problems for the Anderson model. Communications in mathematical physics, 132(3):613-655, 1990.

[GPS17] Patricia Gonçalves, Nicolas Perkowski, and Marielle Simon. Derivation of the stochastic Burgers equation with Dirichlet boundary conditions from the WASEP. 2017.

[Kar85] Mehran Kardar. Depinning by quenched randomness. Physical review letters, 55(21):2235, 1985.

[Kar87] Mehran Kardar. Replica bethe ansatz studies of two-dimensional interfaces with quenched random impurities. Nuclear Physics B, 290:582-602, 1987.

[Kim19] Yujin H Kim. The lower tail of the half-space KPZ equation. arXiv preprint arXiv:1905.07703, 2019.

[KK07] IV Kolokolov and SE Korshunov. Optimal fluctuation approach to a directed polymer in a random medium. Physical Review B, 75(14):140201, 2007.

[KK09] IV Kolokolov and SE Korshunov. Explicit solution of the optimal fluctuation problem for an elastic string in a random medium. Physical Review E, 80(3):031107, 2009.

[KKX17] Davar Khoshnevisan, Kunwoo Kim, and Yimin Xiao. Intermittency and multifractality: A case study via parabolic stochastic pdes. The Annals of Probability, 45(6A):3697-3751, 2017.

[KLD18] Alexandre Krajenbrink and Pierre Le Doussal. Large fluctuations of the KPZ equation in a half-space. SciPost Phys, 5:032, 2018.

[KLD20] Alexandre Krajenbrink and Pierre Le Doussal. Replica Bethe Ansatz solution to the Kardar-Parisi-Zhang equation on the half-line. SciPost Phys., 8, 2020.

[KMS16] Alex Kamenev, Baruch Meerson, and Pavel V Sasorov. Short-time height distribution in the one-dimensional kardarparisi-zhang equation: Starting from a parabola. Physical Review E, 94(3):032108, 2016.

[KPZ86] Mehran Kardar, Giorgio Parisi, and Yi-Cheng Zhang. Dynamic scaling of growing interfaces. Physical Review Letters, 56(9):889, 1986.

[LDMRS16] Pierre Le Doussal, Satya N. Majumdar, Alberto Rosso, and Grégory Schehr. Exact short-time height distribution in the one-dimensional kardar-parisi-zhang equation and edge fermions at high temperature. Phys. Rev. Lett., 117:070403, Aug 2016.

[LDMS16] Pierre Le Doussal, Satya N Majumdar, and Grégory Schehr. Large deviations for the height in 1D Kardar-Parisi-Zhang growth at late times. EPL (Europhysics Letters), 113(6):60004, 2016.

[MKV16] Baruch Meerson, Eytan Katzav, and Arkady Vilenkin. Large deviations of surface height in the kardar-parisi-zhang equation. Physical review letters, 116(7):070601, 2016.

[MS17] Baruch Meerson and Johannes Schmidt. Height distribution tails in the Kardar-Parisi-Zhang equation with Brownian initial conditions. Journal of Statistical Mechanics: Theory and Experiment, 2017(10):103207, 2017.

[MV18] Baruch Meerson and Arkady Vilenkin. Large fluctuations of a Kardar-Parisi-Zhang interface on a half line. Physical Review E, 98(3):032145, 2018.

[Olv97] Frank Olver. Asymptotics and special functions. CRC Press, 1997.

[Par19a] Shalin Parekh. Positive random walks and an identity for half-space SPDEs. arXiv preprint arXiv:1901.09449, 2019.

[Par19b] Shalin Parekh. The KPZ limit of ASEP with boundary. Communications in Mathematical Physics, 365(2):569-649, 2019 .

[Rai00] Eric M Rains. Correlation functions for symmetrized increasing subsequences. arXiv preprint math/0006097, 2000.

[SI04] T Sasamoto and T Imamura. Fluctuations of the one-dimensional polynuclear growth model in half-space. Journal of statistical physics, 115(3-4):749-803, 2004.

[SMP17] Pavel Sasorov, Baruch Meerson, and Sylvain Prolhac. Large deviations of surface height in the 1+ 1-dimensional Kardar-Parisi-Zhang equation: exact long-time results for $\lambda H<0$. Journal of Statistical Mechanics: Theory and Experiment, 2017(6):063203, 2017.

[Tsa18] Li-Cheng Tsai. Exact lower tail large deviations of the KPZ equation. arXiv preprint arXiv:1809.03410, 2018.

[TW96] Craig A Tracy and Harold Widom. On orthogonal and symplectic matrix ensembles. Communications in Mathematical Physics, 177(3):727-754, 1996.

[TW09] Craig A Tracy and Harold Widom. The distributions of random matrix theory and their applications. In New trends in mathematical physics, pages 753-765. Springer, 2009.

[Wu18] Xuan Wu. Intermediate disorder regime for half-space directed polymers. arXiv preprint arXiv:1804.09815, 2018. 
Y. Lin, Department of Mathematics, Columbia University, 2990 Broadway, New York, Ny 10027

E-mail address: y13609@columbia.edu 